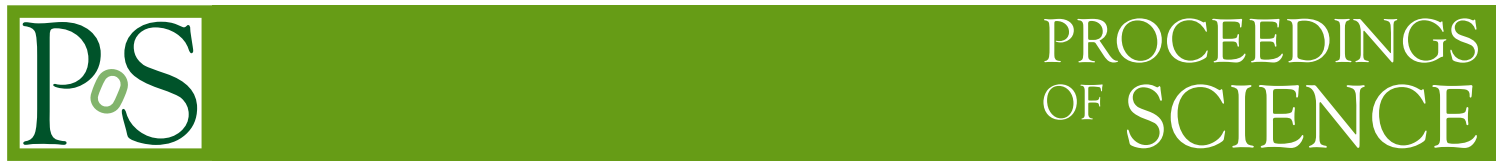

\title{
Astrophysical implications of the Asymptotic Safety Scenario in Quantum Gravity
}

\author{
Alfio Bonanno* \\ INAF, Osservatorio Astrofisico di Catania, Via S.Sofia 78, 95123 Catania, Italy. \\ INFN, Via S.Sofia 64, 95123 Catania, Italy. \\ E-mail: abo@oact.inaf.it
}

In recent years it has emerged that the high energy behavior of gravity could be governed by an ultraviolet non-Gaussian fixed point of the (dimensionless) Newton's constant, whose behavior at high energy is thus antiscreened. This phenomenon has several astrophysical implications. In particular in this article recent works on renormalization group improved cosmologies based upon a renormalization group trajectory of Quantum Einstein Gravity with realistic parameter values will be reviewed. It will be argued that quantum effects can account for the entire entropy of the present Universe in the massless sector and give rise to a phase of inflationary expansion. Moreover, the prediction for the final state of the black hole evaporation, is a Planck size remnant which is formed in an infinite time.

Workshop on Continuum and Lattice Approaches to Quantum Gravity

September 17-192008

Brighton, University of Sussex, United Kingdom

\footnotetext{
${ }^{*}$ Speaker.
} 


\section{Introduction}

Cosmology is a natural setting to study quantum gravity, which may provide answers to fundamental questions as why is the expansion of the universe isotropic, can the initial singularity be avoided, why does the vacuum energy "gravitate" so little (Cosmological Constant problem)?

In recent years it has emerged that the asymptotic safety scenario [1-3] could provide the right framework to address the above questions. According to this approach the ultraviolet (UV) behavior of quantum gravity is controlled by a fixed point at a non-zero value of the (dimensionless) coupling constant, so that the dimensionful Newton's constant reduces its strength at higher energies, it is thus antiscreened. The non-perturbative renormalization group (RG) equation employed in this investigation predicts that the dimensionless cosmological constant reaches a non-gaussian fixed point (NGFP) in the infinite cutoff limit, so that the full Einstein-Hilbert Lagrangian is renormalizable at a non-perturbative level around this fixed point.

The gravitational antiscreening behavior is very similar to the running of the non-Abelian gauge coupling in Yang-Mills Theory, but only after the introduction of the effective average action and its functional renormalization group equation for gravity [4] detailed investigations of the scaling behavior of the Newtons's constant have become possible [4-19]. The non-perturbative renormalization group equation underlying this approach defines a Wilsonian RG flow on a theory space which consists of all diffeomorphism invariant functionals of the metric $g_{\mu \nu}$.

This framework turned out to be an ideal setting for investigating the asymptotic safety scenario in gravity [1-3] and, in fact, substantial evidence was found for the non-perturbative renormalizability of Quantum Einstein Gravity. The theory emerging from this construction ("QEG") is not a quantization of classical general relativity. Instead, its bare action corresponds to a nontrivial fixed point of the RG flow and therefore is a prediction. The effective average action $[4,20]$ has crucial advantages as compared to other continuum implementations of the Wilson RG, in particular it is closely related to the standard effective action and defines a family of effective field theories $\left\{\Gamma_{k}\left[g_{\mu v}\right], 0 \leq k<\infty\right\}$ labeled by the coarse graining scale $k$. The latter property opens the door to a rather direct extraction of physical information from the RG flow, at least in single-scale cases: If the physical process or phenomenon under consideration involves only a single typical momentum scale $p_{0}$ it can be described by a tree-level evaluation of $\Gamma_{k}\left[g_{\mu v}\right]$, with $k=p_{0}$. The precision which can be achieved by this effective field theory description depends on the size of the fluctuations relative to the mean values. If they are large, or if more than one scale is involved, it might be necessary to go beyond the tree analysis.

The qualitative scale dependence of Newton's constant can be grasped with the help of the following physical argument. Let us imagine that in the large distance limit the leading quantum effects of the geometry are described by quantizing the linear fluctuations of the metric, $g_{\mu \nu}$. The resulting theory is a minimallu coupled theory in a curved background spacetime whose elementary quanta, the gravitons, carry energy and momentum. The vacuum of this theory will be populated by virtual graviton pairs, and the problem is to understand how these virtual gravitons respond to the perturbation by an external test body which we immerse in the vacuum. Assuming that also in this situation gravity is universally attractive, the gravitons will be attracted towards the test body. It will thus become "dressed" by a cloud of virtual gravitons surrounding it so that its effective mass seen by a distant observer is larger than it would be in absence of any quantum effects. This 
means that while in QED the quantum fluctuations screen external charges, in quantum gravity they have an antiscreening effect on external test masses. The consequence of this simple Gedanken experiment entails Newton's constant becoming a scale dependent quantity $G(k)$ which is small at small distances $r \sim 1 / k$, and which becomes large at larger distances.

In QED the screening behavior is well-known but it is interesting to recall how this result is obtained from the "renormalization group improvement", a standard device, in particle physics, in order to add the dominant quantum corrections to the Born approximation of a scattering cross section for instance. One starts from the classical potential energy $V_{\mathrm{cl}}(r)=e^{2} / 4 \pi r$ and replaces $e^{2}$ by the running gauge coupling in the one-loop approximation:

$$
e^{2}(k)=e^{2}\left(k_{0}\right)\left[1-b \ln \left(k / k_{0}\right)\right]^{-1}, \quad b \equiv e^{2}\left(k_{0}\right) / 6 \pi^{2} .
$$

The crucial step is to identify the renormalization point $k$ with the inverse of the distance $r$ so that result of this substitution reads

$$
V(r)=-e^{2}\left(r_{0}^{-1}\right)\left[1+b \ln \left(r_{0} / r\right)+O\left(e^{4}\right)\right] / 4 \pi r
$$

where the IR reference scale $r_{0} \equiv 1 / k_{0}$ has to be kept finite in the massless theory. We emphasize that eq.(1.2) is the correct (one-loop, massless) Uehling potential which is usually derived by more conventional perturbative methods [21]. Obviously the position dependent renormalization group improvement $e^{2} \rightarrow e^{2}(k), k \propto 1 / r$ encapsulates the most important effects which the quantum fluctuations have on the electric field produced by a point charge.

The effective field theory techniques proved useful for an understanding of the scale dependent geometry of the effective QEG spacetimes [22-24]. In particular it has been shown [6,22] that these spacetimes have fractal properties, with a fractal dimension of 2 at small, and 4 at large distances. The same dynamical dimensional reduction was also observed in numerical studies of Lorentzian dynamical triangulations [25-27] and in [28] A.Connes et al. speculated about its possible relevance to the non-commutative geometry of the standard model.

In order to extract all the relevant information from the RG evolution, it is thus necessary to relate the cutoff scale $k$ which corresponds to the resolution of the RG flow, to the spacetime properties. This procedure is called "cutoff identification" for which the relevant energy scale $k$ is related to a characteristic length scale where the quanta with energy $k$ propagate. In the case of massless QED the choice $k \propto 1 / r$ was clearly the only possible one, as there are no other relevant scales in the problem. When several scales are present the prescription which emerges from the general theory of the Effective Average Action [20] is that $\Gamma_{k}$ is defined at a scale $k$ which is the largest one of the various competing scales in the fluctuation determinant of the Average Action, $\Gamma_{k}^{(2)}$, namely

$$
\Gamma_{k}^{(2)}=\frac{\delta^{2} \Gamma_{k}}{\delta \Phi^{2}}
$$

where $\Phi$ is the so-called "blocked" field [29].

The difficulty arises when we decide to apply the same "recipe" in gravity by writing

$$
\left.k \sim 1 / \ell\left(x^{\mu}\right)\right), \quad \ell=\ell\left(g_{\mu v}\right)
$$

being $\ell$ a characteristic length where the fluctuations with energy $k$ propagate. The reason is that the flow equation is by construction diffeomorphism invariant at any $k$ so that the RG flow itself 
does not know anything about the background field metric $g_{\mu \nu}$ that has been used for projecting on a finite-dimensional subspace of the "theory space".

There are two possible strategies to overcome this issue. The first one amounts to choose a fiducial metric which is a solution of the Einstein equations and RG-improve it by substituting the Newton constant $G$ with the running $G(k)$ together with a cutoff identification of the type (1.4). The limitation of this approach lies in the fact that in general the improved metric may not be a solution of Einstein equation, but one can imagine that this is a sort of "Thomas-Fermi" approximation where only the leading quantum corrections are taken into account $[30,31]$. The improved $g_{\mu \nu}(k)$ metric represents then a sort of "emergent" spacetime description of the effective geometry [32-34] according to the scale dependence of the Newton constant.

A second possibility is to consider the energy scale $k$ associated to the field strength itself rather than to an observational scale $\ell$. This is motivated by the analogy with the QED (and QCD) case, where higher loop contributions to the Uehling potential are obtained by renormalization group improvement of the QED action by using the field strength $\left(F_{\mu \nu} F^{\mu v}\right)^{1 / 4}$ as a cutoff instead than $1 / r$ [35-37]. In this case the short distance correction to the static potential is obtained from the non-linear differential equations

$$
\begin{aligned}
& \nabla \cdot \mathbf{D}=J_{0}, \quad \mathbf{D}=\mathbf{E} \varepsilon(E), \quad \mathbf{E}=-\nabla A^{0} \\
& \varepsilon(E)=1-\frac{e^{2}}{12 \pi^{2}} \log \left(e E / k_{0}^{2}\right)+\ldots
\end{aligned}
$$

whose solution reproduces the Uehling potential in the long distance limit, but in general the solutions of Eq.(1.5) include higher loop effects due to the non-linearities of the effective action in the short distance limit.

The two approaches discussed above are obviously related, at least in some limit. In the case of Robertson-Walker spaces it will be shown that due to the very high degree of symmetry of the spacetime, the time-scale defined by "Hubble parameter" behaves essentially like the characteristic time scale associated to the relevant curvature invariants [38-40]. In the case of spherically symmetric spacetimes [30,31,41,42], near the singularity the proper distance of a radially free falling observer behaves essentially as $1 / \sqrt{\Psi_{2}}$, being $\Psi_{2}$ the "Coulombian" component of the Weyl tensor.

From the above discussion it is then clear that in general there is not a preferred strategy to perform the RG improvement in gravity. In some case it might be more interesting to RG improve solutions and to make contact with an emergent spacetime description of the effective geometry. In some other cases it could be more convenient to work with a RG improvement at the level of field equations or actions [42-45].

It is important to remark that it is not surprising that different cutoff might provide quantitavely different evolutions, as usually the $\beta$-functions are not "universal" quantities. For instance it is well known [46] that different realizations of the block-spin RG transformation applied to the Ising model may provide different values for fixed points, as we are essentially using different type of "microscopes". On the other hand truly universal quantities, like the critical exponents, are essentially insensitive to the cutoff choice.

\footnotetext{
${ }^{1}$ From this point of view the criticism expressed in [47] should not be seriously considered.
} 
In this review recent results obtained with the RG improvement of Einstein theory will be discussed in the framework QEG will be reviewed, with particular emphasis on recent results obtained in cosmology [40]. In particular in Sec.2 the RG evolution of the Newton constant and Cosmological constant describing our Universe are described. In Sec.3 a covariant formalism to improve the Einstein field equation is presented while in Sec.4 the RG improved Robertson-Walker Cosmology is discussed. In Sec.5 the basic mechanism to produce the entropy of the Universe is presented. In Sec. 6 the properties of a class of solutions of the RG equations are discussed. In Sec.7 a mechanism to produce a power-law inflation is studied. In Sec.8 and Sec.9 the properties of RG improved Black Hole metric is studied. In Sec.10 the possibility that Quantum Gravity effects are present on Astrophysical distances is reviewed. Sec.11 is devoted to the Conclusions.

\section{The RG trajectory of our Universe}

It is possible to show that there exists a class of RG trajectories obtained from QEG in the Einstein-Hilbert approximation [4], namely those of the "Type IIIa" [9] which possesses all the qualitative properties one would expect from the RG trajectory describing gravitational phenomena in the real Universe we live in. In particular they can have a long classical regime and a small, positive cosmological constant in the infrared. Determining its parameters from observations, one finds [40] that, according to this particular QEG trajectory, the running cosmological constant $\Lambda(k)$ changes by about 120 orders of magnitude between $k$-values of the order of the Planck mass and macroscopic scales, while the running Newton constant $G(k)$ has no strong $k$-dependence in this regime. For $k>m_{\mathrm{Pl}}$, the non-Gaussian fixed point which is responsible for the renormalizability of QEG controls their scale dependence. In the deep ultraviolet $(k \rightarrow \infty), \Lambda(k)$ diverges and $G(k)$ approaches zero.

Is there any experimental or observational evidence that would hint at this enormous scale dependence of the gravitational parameters, the cosmological constant in particular? As it was stressed before, even though it is always difficult to give a precise physical interpretation to the RG scale $k$ it is fairly certain that any sensible identification of $k$ in terms of cosmological quantities will lead to a $k$ which decreases during the expansion of the Universe. As a consequence, $\Lambda(k)$ will also decrease as the Universe expands. Already the purely qualitative assumption of a positive and decreasing cosmological constant supplies an interesting hint as to which phenomena might reflect a possible $\Lambda$-running.

To make the argument as simple as possible, let us first consider a Universe without matter, but with a positive $\Lambda$. Assuming maximal symmetry, this is nothing but de Sitter space, of course. In static coordinates its metric is $d s^{2}=-\left(1+2 \Phi_{\mathrm{N}}(r)\right) d t^{2}+\left(1+2 \Phi_{\mathrm{N}}(r)\right)^{-1} d r^{2}+r^{2}\left(d \theta^{2}+\sin ^{2} \theta d \phi^{2}\right)$ with $\Phi_{\mathrm{N}}(r)=-\frac{1}{6} \Lambda r^{2}$. In the weak field and slow motion limit $\Phi_{\mathrm{N}}$ has the interpretation of a Newtonian potential, with a correspondingly simple physical interpretation. The left panel of Fig. 1 shows $\Phi_{N}$ as a function of $r$; for $\Lambda>0$ it is an upside-down parabola. Point particles in this spacetime, symbolized by the black dot in Fig.1, "roll down the hill" and are rapidly driven away from the origin and from any other particle. Now assume that the magnitude of $|\Lambda|$ is slowly ("adiabatically") decreased. This will cause the potential $\Phi_{\mathrm{N}}(r)$ to move upward as a whole, its slope decreases. So the change in $\Lambda$ increases the particle's potential energy. This is the simplest way of understanding that a positive decreasing cosmological constant has the effect of "pumping" 


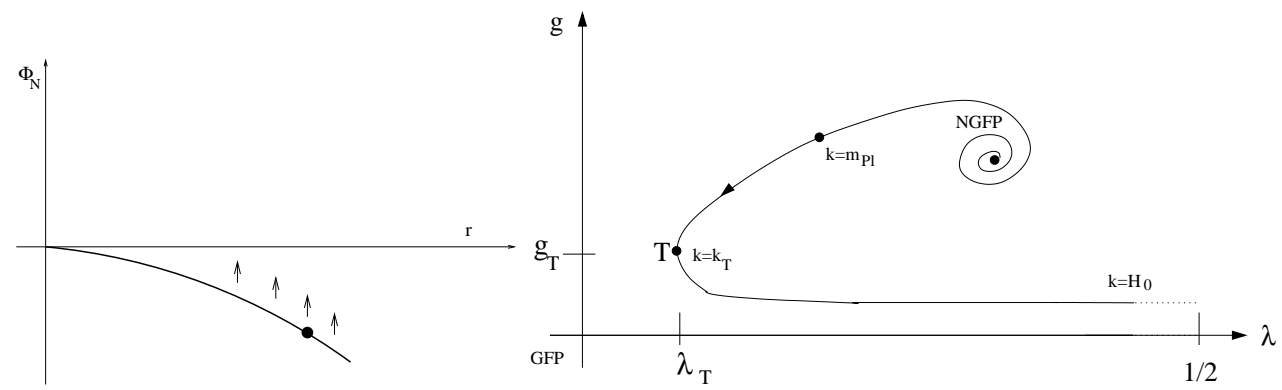

Figure 1: The left panel shows the quasi-Newtonian potential corresponding to de Sitter space. The curve moves upward as the cosmological constant decreases. On the right panel the "realistic" RG trajectory.

energy into the matter degrees of freedom. More realistically one will describe the matter system in a hydrodynamics or quantum field theory language and one will include its backreaction onto the metric. But the basic conclusion, namely that a slow decrease of a positive $\Lambda$ transfers energy into the matter system, will remain true.

We are thus led to suspect that, because of the decreasing cosmological constant, there is a continuous inflow of energy into the cosmological fluid contained in an expanding Universe. It will "heat up" the fluid or, more exactly, lead to a slower decrease of the temperature than in standard cosmology. Furthermore, by elementary thermodynamics, it will increase the entropy of the fluid. If during the time $d t$ an amount of heat $d Q>0$ is transferred into a volume $V$ at the temperature $T$ the entropy changes by an amount $d S=d Q / T>0$. To be as conservative (i.e., close to standard cosmology) as possible, we assume that this process is reversible. If not, $d S$ is even larger.

In standard Friedmann-Robertson-Walker (FRW) cosmology the expansion is adiabatic, the entropy (within a comoving volume) is constant. It has always been somewhat puzzling therefore where the huge amount of entropy contained in the present Universe comes from. Presumably it is dominated by the CMBR photons which contribute an amount of about $10^{88}$ to the entropy within the present Hubble sphere. (We use units such that $k_{\mathrm{B}}=1$. ) In fact, if it true that no entropy is produced during the expansion then the Universe would have had an entropy of at least $10^{88}$ immediately after the initial singularity which for various reasons seems quite unnatural. In scenarios which invoke a "tunneling from nothing", for instance, spacetime was "born" in a pure quantum state, so the very early Universe is expected to have essentially no entropy. Usually it is argued that the present entropy is the result of some sort of "coarse graining" which, however, typically is not considered as an active part of the cosmological dynamics in the sense that it would have an impact on the time evolution of the metric.

In [40] it was argued that in principle the entire entropy of the massless fields in the present universe can be understood as arising from the mechanism described above. If energy can be exchanged freely between the cosmological constant and the matter degrees of freedom, the entropy observed today is obtained precisely if the initial entropy at the "big bang" vanishes. The assumption that the matter system must allow for an unhindered energy exchange with $\Lambda$ is essential.

There is another, more direct potential consequence of a decreasing positive cosmological constant, namely a period of automatic inflation during the very first stages of the cosmological evolution. It is not surprising, of course, that a positive $\Lambda$ can cause an accelerated expansion, but 
in the classical context the problem with a $\Lambda$-driven inflation is that it would never terminate once it has started. In popular models of scalar driven inflation this problem is circumvented by designing the inflaton potential in such a way that it gives rise to a vanishing vacuum energy after a period of "slow roll".

There will thus be reviewed generic RG cosmologies based upon the QEG trajectories which have an era of $\Lambda$-driven inflation immediately after the big bang which ends automatically as a consequence of the RG running of $\Lambda(k)$. Once the scale $k$ drops significantly below $m_{\mathrm{Pl}}$, the accelerated expansion ends because the vacuum energy density $\rho_{\Lambda}$ is already too small to compete with the matter density. Clearly this is a very attractive scenario: no ad hoc ingredients such as an inflaton field or a special potential are needed to trigger inflation. It suffices to include the leading quantum effects in the gravity + matter system. Furthermore, it will be shown that asymptotic safety offers a natural mechanism for the quantum mechanical generation of primordial density perturbations, the seeds of cosmological structure formations.

\section{A covariant approach to RG improvement in cosmology}

In the following we shall present the improved RG equation in the $3+1$ formalism. Let $g_{\mu \nu}$ be the space-time metric with signature $(-,+,+,+)$. A "cosmological fundamental observer" comoving with the cosmological fluid has 4-velocity $u^{\mu}=d x^{\mu} / d \tau$ with $u^{\mu} u_{\mu}=-1$, where $\tau$ is the proper time along the fluid flow lines. The projection tensor onto the tangent 3-space orthogonal to $u^{\mu}$ is $h_{\mu v}=g_{\mu v}+u_{\mu} u_{v}$, with $h^{\mu}{ }_{v} h_{\sigma}=h^{\mu}{ }_{\sigma}$ and $h^{\mu}{ }_{v} u^{v}=0$. We denote by a semicolon the standard covariant derivative and by an over-dot the differentiation with respect to the proper time $\tau$. The covariant derivative of $u^{\mu}$ reads as

$$
u_{\mu ; v}=\omega_{\mu v}+\sigma_{\mu v}+\frac{1}{3} \Theta h_{\mu v}-\dot{u}_{\mu} u_{v}
$$

where $\omega_{\mu v}=h^{\alpha}{ }_{\mu} h^{\beta}{ }_{\nu} u_{[\alpha ; \beta]}$ is the vorticity tensor, $\sigma_{\mu v}=h^{\alpha}{ }_{\mu} h^{\beta}{ }_{v} u_{(\alpha ; \beta)}-\frac{1}{3} \Theta h_{\mu v}$ is the shear tensor, $\Theta=u_{; \mu}^{\mu}$ is the expansion scalar and $\dot{u}^{\mu}=u_{; v}^{\mu} u^{v}$ is the acceleration four-vector; square and round brackets denote anti-symmetrization and symmetrization, respectively.

One can introduce a representative length $\ell$ along the particle world-lines by the equation

$$
\dot{\ell} / \ell=\frac{1}{3} \Theta
$$

In fact $\ell$ represent completely the volume behavior of the fluid as any comoving volume element is proportional to $\ell^{3}$. The net effect of $\Theta$ is in fact to change fluid sphere into another fluid sphere with the the same orientation but with different volume. One can then define the "Hubble parameter" and the deceleration parameter by

$$
H \equiv \dot{\ell} / \ell, \quad q \equiv \ddot{\ell} / \ell H^{2}
$$

The Einstein equations read

$$
R_{\mu v} u^{\mu} u^{v}=4 \pi G(\rho+3 p)-\Lambda, \quad R_{\alpha \beta} h_{\mu}^{\alpha} h_{v}^{\beta}=[4 \pi G(\rho-p)+\Lambda]
$$


where $\Lambda=\Lambda\left(x^{\mu}\right)$ is the position-dependent cosmological term and $G=G\left(x^{\mu}\right)$ the position-dependent Newton parameter. The energy-momentum tensor is assumed to have the the perfect fluid form $T^{\mu v}=(p+\rho) u^{\mu} u^{v}+p g^{\mu v}$. The Bianchi identities lead to the conservation law along $u^{\mu}$

$$
\dot{\rho}+\Theta(\rho+p)=-\frac{1}{8 \pi G}[8 \pi \dot{G} \rho+\dot{\Lambda}]
$$

and onto the orthogonal hypersurface

$$
\dot{u}^{\mu}+\frac{h^{\mu v} p_{; v}}{\rho+p}=\frac{h^{\mu v}}{8 \pi G}\left[\Lambda_{, v}-8 \pi p G_{, v}\right]
$$

The Raychaudhuri equation is obtained with the help of the Einstein field equations and of Eq. (3.1),

$$
\dot{\Theta}+\frac{1}{3} \Theta^{2}+2\left(\sigma^{2}-\omega^{2}\right)-\dot{u}_{; \mu}^{\mu}+4 \pi G(\rho+3 p)-\Lambda=0,
$$

where $2 \sigma^{2} \equiv \sigma_{\mu \nu} \sigma^{\mu \nu}$ and $2 \omega^{2} \equiv \omega_{\mu v} \omega^{\mu \nu}$. The scalar curvature of the tangent space is given by

$$
\mathscr{K} \equiv{ }^{(3)} R=R+2 R_{\mu v} u^{\mu} u^{v}+2 \sigma^{2}-2 \omega^{2}-\frac{2}{3} \Theta^{2},
$$

which leads, by using the field equations (3.4), to the generalized Friedmann equation

$$
\mathscr{K}=2 \sigma^{2}-2 \omega^{2}-\frac{2}{3} \Theta^{2}+16 \pi G \rho+2 \Lambda .
$$

In homogeneous spaces, Eq.(3.6) is identically satisfied, while Eq.(3.9) reduces to the familiar Friedmann equation which is coupled to the energy balance equation (3.5). In order to integrate the previous equations in a general spacetime, the evolution equations for shear and vorticity are needed, together with the dynamical equations for $G$ and $\Lambda$ which are obtained by the RG equations. The latter are obtained in the Einstein-Hilbert truncation as a set of $\beta$-functions for the dimensionless Newton constant and cosmological constant, $g$ and $\lambda$,

$$
k \partial_{k} g=\beta_{g}(g, \lambda), \quad k \partial_{k} \lambda=\beta_{\lambda}(g, \lambda),
$$

and the link with the spacetime dynamics is provided by the cut-off identification

$$
k=k(\tau, \rho, \dot{\rho}, \Theta, \dot{\Theta}, \ldots)
$$

The dots stand for all possible physical or geometrical invariants which can act as IR regulators in the fluctuation determinant of $\Gamma_{k}$. The knowledge of the precise functional dependence in Eq. (3.11) would then provide a dynamical evolution which is consistent with the full effective action at $k=0$. In Ref. [48] the simple choice $k \propto 1 / t$ can be justified on the ground that, if there are no other scales in the system when the Universe had age $t$, fluctuations with frequency greater than $1 / t$ may not have played any role as yet, and the running must be stopped at $k \propto 1 / t$. On the other hand, recent works [38-40] have argued that the Hubble parameter defined in Eq.(3.3) is a physically meaningful cutoff as it measures the curvature of the spacetime, and it also reproduces the $1 / t$ cutoff for any power-law dependence of $\ell(t)$ in Eq.(3.2). In fact the scalar curvature $R$, the 
square of the Ricci tensor $\mathscr{R}=R_{\alpha \beta} R^{\alpha \beta}$ and the Kretschmann invariant $K=R_{\alpha \beta \gamma \delta} R^{\alpha \beta \gamma \delta}$ can all be expressed in terms of the Hubble parameter and its derivatives,

$$
R=6\left(2 H^{2}+\dot{H}\right), \quad \mathscr{R}=12\left(3 H^{4}+3 H^{2} \dot{H}+\dot{H}^{2}\right), \quad K=12\left(2 H^{4}+2 H^{2} \dot{H}+\dot{H}^{2}\right)
$$

On the other hand, the second functional derivative of the effective average action reads [14] $\Gamma_{k}^{(2)}[g, g]_{\rho \sigma}^{\mu v}=2 \kappa^{2} Z_{N k}\left[-K_{\rho \sigma}^{\mu v} D^{2}+U_{\rho \sigma}^{\mu v}\right]$ where

$$
K_{\rho \sigma}^{\mu v}=\frac{1}{4}\left[\delta_{\rho}^{\mu} \delta_{\sigma}^{v}+\delta_{\sigma}^{\mu} \delta_{\rho}^{v}-g^{\mu v} g_{\rho \sigma}\right]
$$

and

$$
\begin{aligned}
& U_{\rho \sigma}^{\mu v}=\frac{1}{4}\left[\delta_{\rho}^{\mu} \delta_{\sigma}^{v}+\delta_{\sigma}^{\mu} \delta_{\rho}^{v}-g^{\mu v} g_{\rho \sigma}\right]\left(R-2 \bar{\lambda}_{k}\right)+\frac{1}{2}\left[g^{\mu v} R_{\rho \sigma}+g_{\rho \sigma} R^{\mu v}\right] \\
& -\frac{1}{4}\left[\delta_{\rho}^{\mu} R_{\sigma}^{v}+\delta_{\sigma}^{\mu} R_{\rho}^{v}+\delta_{\rho}^{v} R_{\sigma}^{\mu}+\delta_{\sigma}^{v} R_{\rho}^{\mu}\right]-\frac{1}{2}\left[R^{v}{ }_{\rho}^{\mu}{ }_{\sigma}+R^{v}{ }_{\sigma}^{\mu}{ }_{\rho}\right]
\end{aligned}
$$

which clearly shows the Ricci scalar, the Ricci tensor and the Riemann tensor enters in the fluctuation determinant as mass-type regulators. As from (3.12) all those terms are essentially expressible in terms of the Hubble parameter, it is then clear that one can conveniently parameterize the field strength dependence in terms of the single scalar $H$. It is important to stress that this is a consequence of the very high degree of symmetry of our spacetime, but in a generic spacetime the actual cutoff can be different.

\section{The improved Robertson-Walker cosmology}

Let us now specify our spacetime to describe a spatially flat $(K=0)$ Robertson-Walker metric with scale factor $\ell(t)$, so that the shear, rotation and acceleration are identically vanishing. We can take $T_{\mu}{ }^{v}=\operatorname{diag}[-\rho, p, p, p]$ to be the energy momentum tensor of an ideal fluid with equation of state $p=w \rho$ where $w>-1$ is constant.

Then the improved Einstein equation boils down to the modified Friedmann equation and a continuity equation:

$$
\begin{aligned}
& H^{2}=\frac{8 \pi}{3} G(t) \rho+\frac{1}{3} \Lambda(t) \\
& \dot{\rho}+3 H(\rho+p)=-\frac{\dot{\Lambda}+8 \pi \rho \dot{G}}{8 \pi G}
\end{aligned}
$$

The modified continuity equation above is the integrability condition for the improved Einstein equation implied by Bianchi's identity, $D^{\mu}\left[-\Lambda(t) g_{\mu \nu}+8 \pi G(t) T_{\mu v}\right]=0$. It describes the energy exchange between the matter and gravitational degrees of freedom (geometry). For later use let us note that upon defining the critical density $\rho_{\text {crit }}(t) \equiv 3 H(t)^{2} / 8 \pi G(t)$ and the relative densities $\Omega_{\mathrm{M}} \equiv \rho / \rho_{\text {crit }}$ and $\Omega_{\Lambda}=\rho_{\Lambda} / \rho_{\text {crit }}$ the modified Friedmann equation (4.1) can be written as $\Omega_{\mathrm{M}}(t)+$ $\Omega_{\Lambda}(t)=1$.

It is possible to obtain $G(k)$ and $\Lambda(k)$ by solving the flow equation in the Einstein-Hilbert truncation with a sharp cutoff $[4,39]$. It is formulated in terms of the dimensionless Newton and 
cosmological constant, respectively: $g(k) \equiv k^{2} G(k), \lambda(k)=\Lambda(k) / k^{2}$. Quantum corrected cosmologies are computed by (numerically) solving the RG improved evolution equations. The cutoff identification

$$
k(t)=\xi H(t)
$$

where $\xi$ is a fixed positive constant of order unity will be employed. As discussed in the previous section this is a natural choice since in a Robertson-Walker geometry the Hubble parameter measures the curvature of spacetime which is related to the actual regulator. Thus we have

$$
G(t)=\frac{g(\xi H(t))}{\xi^{2} H(t)^{2}}, \quad \Lambda(t)=\xi^{2} H(t)^{2} \lambda(\xi H(t))
$$

Let us briefly review how the type IIIa trajectories of the Einstein-Hilbert truncation can be matched against the observational data. This analysis is fairly robust and clearcut; it does not involve the NGFP. All that is needed is the RG flow linearized about the Gaussian fixed point (GFP) which is located at $g=\lambda=0$. In its vicinity one has [40] $\Lambda(k)=\Lambda_{0}+v \bar{G} k^{4}+\cdots$ and $G(k)=\bar{G}+\cdots$. Or, in terms of the dimensionless couplings, $\lambda(k)=\Lambda_{0} / k^{2}+v \bar{G} k^{2}+\cdots, g(k)=$ $\bar{G} k^{2}+\cdots$. In the linear regime of the GFP, $\Lambda$ displays a running $\propto k^{4}$ and $G$ is approximately constant. Here $v$ is a positive constant of order unity [39], $v \equiv \frac{1}{4 \pi} \Phi_{2}^{1}(0)$. These equations are valid if $\lambda(k) \ll 1$ and $g(k) \ll 1$. They describe a 2-parameter family of RG trajectories labeled by the pair $\left(\Lambda_{0}, \bar{G}\right)$. It will prove convenient to use an alternative labeling $\left(\lambda_{\mathrm{T}}, k_{\mathrm{T}}\right)$ with $\lambda_{\mathrm{T}} \equiv\left(4 v \Lambda_{0} \bar{G}\right)^{1 / 2}$ and $k_{\mathrm{T}} \equiv\left(\Lambda_{0} / \nu \bar{G}\right)^{1 / 4}$. The old labels are expressed in terms of the new ones as $\Lambda_{0}=\frac{1}{2} \lambda_{\mathrm{T}} k_{\mathrm{T}}^{2}$ and $\bar{G}=\lambda_{\mathrm{T}} / 2 v k_{\mathrm{T}}^{2}$. It is furthermore convenient to introduce the abbreviation $g_{\mathrm{T}} \equiv \lambda_{\mathrm{T}} / 2 v$.

When parameterized by the pair $\left(\lambda_{\mathrm{T}}, k_{\mathrm{T}}\right)$ the trajectories assume the form

$$
\begin{aligned}
& \Lambda(k)=\frac{1}{2} \lambda_{\mathrm{T}} k_{\mathrm{T}}^{2}\left[1+\left(k / k_{\mathrm{T}}\right)^{4}\right] \equiv \Lambda_{0}\left[1+\left(k / k_{\mathrm{T}}\right)^{4}\right] \\
& G(k)=\frac{\lambda_{\mathrm{T}}}{2 v k_{\mathrm{T}}^{2}} \equiv \frac{g_{\mathrm{T}}}{k_{\mathrm{T}}^{2}}
\end{aligned}
$$

or, in dimensionless form,

$$
\lambda(k)=\frac{1}{2} \lambda_{\mathrm{T}}\left[\left(\frac{k_{\mathrm{T}}}{k}\right)^{2}+\left(\frac{k}{k_{\mathrm{T}}}\right)^{2}\right], \quad g(k)=g_{\mathrm{T}}\left(\frac{k}{k_{\mathrm{T}}}\right)^{2}
$$

As for the interpretation of the new variables, it is clear that $\lambda_{\mathrm{T}} \equiv \lambda\left(k \equiv k_{\mathrm{T}}\right)$ and $g_{\mathrm{T}} \equiv g(k=$ $k_{\mathrm{T}}$ ), while $k_{\mathrm{T}}$ is the scale at which $\beta_{\lambda}$ (but not $\beta_{g}$ ) vanishes according to the linearized running: $\beta_{\lambda}\left(k_{\mathrm{T}}\right) \equiv k d \lambda(k) /\left.d k\right|_{k=k_{\mathrm{T}}}=0$. Thus we see that $\left(g_{\mathrm{T}}, \lambda_{\mathrm{T}}\right)$ are the coordinates of the turning point $\mathrm{T}$ of the type IIIa trajectory considered, and $k_{\mathrm{T}}$ is the scale at which it is passed. It is convenient to refer the "RG time" $\tau$ to this scale: $\tau(k) \equiv \ln \left(k / k_{\mathrm{T}}\right)$. Hence $\tau>0(\tau<0)$ corresponds to the "UV regime" ("IR regime") where $k>k_{\mathrm{T}}\left(k<k_{\mathrm{T}}\right)$.

Let us now hypothesize that, within a certain range of $k$-values, the RG trajectory realized in Nature can be approximated by (4.6). In order to determine its parameters $\left(\Lambda_{0}, \bar{G}\right)$ or $\left(\lambda_{\mathrm{T}}, k_{\mathrm{T}}\right)$ we must perform a measurement of $G$ and $\Lambda$. If we interpret the observed values $G_{\text {observed }}=m_{\mathrm{Pl}}^{-2}, m_{\mathrm{Pl}} \approx$ $1.2 \times 10^{19} \mathrm{GeV}$, and $\Lambda_{\text {observed }}=3 \Omega_{\Lambda 0} H_{0}^{2} \approx 10^{-120} m_{\mathrm{Pl}}^{2}$ as the running $G(k)$ and $\Lambda(k)$ evaluated at a scale $k \ll k_{\mathrm{T}}$, then we get from (4.5) that $\Lambda_{0}=\Lambda_{\text {observed }}$ and $\bar{G}=G_{\text {observed. }}$ Using the definitions of $\lambda_{\mathrm{T}}$ and $k_{\mathrm{T}}$ along with $v=O(1)$ this leads to the order-of-magnitude estimates $g_{\mathrm{T}} \approx \lambda_{\mathrm{T}} \approx 10^{-60}$ 
and $k_{\mathrm{T}} \approx 10^{-30} m_{\mathrm{Pl}} \approx\left(10^{-3} \mathrm{~cm}\right)^{-1}$. Because of the tiny values of $g_{\mathrm{T}}$ and $\lambda_{\mathrm{T}}$ the turning point lies in the linear regime of the GFP.

Up to this point we discussed only that segment of the "trajectory realized in Nature" which lies inside the linear regime of the GFP. The complete RG trajectory is obtained by continuing this segment with the flow equation both into the IR and into the UV, where it ultimately spirals into the NGFP. While the UV-continuation is possible within the Einstein-Hilbert truncation, this approximation breaks down in the IR when $\lambda(k)$ approaches $1 / 2$. Interestingly enough, this happens near $k=H_{0}$, the present Hubble scale. The right panel of Fig.1 shows a schematic sketch of the complete trajectory on the $g$ - $\lambda$-plane and Fig. 2 displays the resulting $k$-dependence of $G$ and $\Lambda$.

\section{Primordial entropy generation}

Let us return to the modified continuity equation (3.5). After multiplication by $a^{3}$ it reads

$$
[\dot{\rho}+3 H(\rho+p)] a^{3}=\widetilde{\mathscr{P}}(t)
$$

where we defined

$$
\widetilde{\mathscr{P}} \equiv-\left(\frac{\dot{\Lambda}+8 \pi \rho \dot{G}}{8 \pi G}\right) a^{3}
$$

Without assuming any particular equation of state eq.(5.1) can be rewritten as

$$
\frac{d}{d t}\left(\rho a^{3}\right)+p \frac{d}{d t}\left(a^{3}\right)=\widetilde{\mathscr{P}}(t)
$$

The interpretation of this equation is as follows. Let us consider a unit coordinate, i.e. comoving volume in the Robertson-Walker spacetime. Its corresponding proper volume is $V=a^{3}$ and its energy contents is $U=\rho a^{3}$. The rate of change of these quantities is subject to (5.3):

$$
\frac{d U}{d t}+p \frac{d V}{d t}=\widetilde{\mathscr{P}}(t)
$$

In classical cosmology where $\widetilde{P} \equiv 0$ this equation together with the standard thermodynamic relation $d U+p d V=T d S$ is used to conclude that the expansion of the Universe is adiabatic, i.e. the entropy inside a comoving volume does not change as the Universe expands, $d S / d t=0$.

Here and in the following we write $S \equiv s a^{3}$ for the entropy carried by the matter inside a unit comoving volume and $s$ for the corresponding proper entropy density.

When $\Lambda$ and $G$ are time dependent, $\widetilde{\mathscr{P}}$ is nonzero and we interpret (5.4) as describing the process of energy (or "heat") exchange between the scalar fields $\Lambda$ and $G$ and the ordinary matter. This interaction causes $S$ to change:

$$
T \frac{d S}{d t}=T \frac{d}{d t}\left(s a^{3}\right)=\widetilde{\mathscr{P}}(t)
$$

The actual rate of change of the comoving entropy is

$$
\frac{d S}{d t}=\frac{d}{d t}\left(s a^{3}\right)=\mathscr{P}(t)
$$

where

$$
\mathscr{P} \equiv \widetilde{\mathscr{P}} / T
$$


If $T$ is known as a function of $t$ we can integrate (5.5) to obtain $S=S(t)$. In the RG improved cosmologies the entropy production rate per comoving volume

$$
\mathscr{P}(t)=-\left[\frac{\dot{\Lambda}+8 \pi \rho \dot{G}}{8 \pi G}\right] \frac{a^{3}}{T}
$$

is nonzero because the gravitational "constants" $\Lambda$ and $G$ have acquired a time dependence.

Clearly we can convert the heat exchanged, $T d S$, to an entropy change only if the dependence of the temperature $T$ on the other thermodynamical quantities, in particular $\rho$ and $p$ is known. For this reason we shall now make the following assumption about the matter system and its (nonequilibrium!) thermodynamics:

The matter system is assumed to consist of $n_{\mathrm{eff}}=n_{\mathrm{b}}+\frac{7}{8} n_{\mathrm{f}}$ species of effectively massless degrees of freedom which all have the same temperature $T$. The equation of state is $p=\rho / 3$, i.e. $w=1 / 3$, and $\rho$ depends on $T$ as

$$
\rho(T)=\kappa^{4} T^{4}, \quad \kappa \equiv\left(\pi^{2} n_{\mathrm{eff}} / 30\right)^{1 / 4}
$$

No assumption is made about the relation $s=s(T)$.

The first assumption, radiation dominance and equal temperature, is plausible since we shall find that there is no significant entropy production any more once $H(t)$ has dropped substantially below $m_{\mathrm{Pl}}$. The second assumption, eq.(5.9), refer to the hypothesis that the injection of energy into the matter system disturbs its equilibrium only very weakly. The approximation is that the equilibrium relations among $\rho, p$, and $T$ are still valid in the non-equilibrium situation of a cosmology with entropy production.

By inserting $p=\rho / 3$ and (5.9) into the modified continuity equation the entropy production rate can be seen to be a total time derivative: $\mathscr{P}(t)=\frac{d}{d t}\left[\frac{4}{3} \kappa a^{3} \rho^{3 / 4}\right]$. Therefore we can immediately integrate (5.6) and obtain $S(t)=\frac{4}{3} \kappa a^{3} \rho^{3 / 4}+S_{\mathrm{c}}, s(t)=\frac{4}{3} \kappa \rho(t)^{3 / 4}+\frac{S_{\mathrm{c}}}{a(t)^{3}}$. Here $S_{\mathrm{c}}$ is a constant of integration. In terms of $T$, using (5.9) again,

$$
s(t)=\frac{2 \pi^{2}}{45} n_{\mathrm{eff}} T(t)^{3}+\frac{S_{\mathrm{c}}}{a(t)^{3}}
$$

The final result (5.10) is very remarkable for at least two reasons. Firstly, for $S_{\mathrm{c}}=0$, eq.(5.10) has exactly the form valid for radiation in equilibrium. Note that we did not postulate this relationship, only the $\rho(T)$-law was assumed. The equilibrium formula $s \propto T^{3}$ was derived from the cosmological equations, i.e. the modified conservation law. This result makes the hypothesis "non-adiabatic, but as little as possible" selfconsistent.

Secondly, if $\lim _{t \rightarrow 0} a(t) \rho(t)^{1 / 4}=0$, which is actually the case for the most interesting class of cosmologies we shall find, then $S(t \rightarrow 0)=S_{c}$. As we mentioned in the introduction, the most plausible initial value of $S$ is $S=0$ which means a vanishing constant of integration $S_{c}$ here. But then, with $S_{c}=0$ the entire entropy carried by the massless degrees of freedom is due to the RG running. So it indeed seems to be true that the entropy of the CMBR photons we observe today is due to a coarse graining. Unexpectedly, not a coarse graining of the matter degrees of freedom but rather of the gravitational ones which determines the background spacetime the photons propagate on. 


\section{Solving the RG improved Einstein Equations}

In [40] the improved Einstein equations $(4.1,4.2)$ have been solved for the trajectory with realistic parameter values which was discussed in Section 3. The solutions were determined by applying the algorithm described at the end of Section 2. Having fixed the RG trajectory, there exists a 1-parameter family of solutions $(H(t), \rho(t))$. This parameter is conveniently chosen to be the relative vacuum energy density in the fixed point regime, $\Omega_{\Lambda}^{*}$.

The very early part of the cosmology can be described analytically. For $k \rightarrow \infty$ the trajectory approaches the NGFP, $(g, \lambda) \rightarrow\left(g_{*}, \lambda_{*}\right)$, so that $G(k)=g_{*} / k^{2}$ and $\Lambda(k)=\lambda_{*} k^{2}$. In this case the differential equation can be solved analytically, with the result

$$
H(t)=\alpha / t, \quad a(t)=A t^{\alpha}, \quad \alpha=\left[\frac{1}{2}(3+3 w)\left(1-\Omega_{\Lambda}^{*}\right)\right]^{-1}
$$

and $\rho(t)=\widehat{\rho} t^{-4}, G(t)=\widehat{G} t^{2}, \Lambda(t)=\widehat{\Lambda} / t^{2}$. Here $A, \widehat{\rho}, \widehat{G}$, and $\widehat{\Lambda}$ are positive constants. They depend on $\Omega_{\Lambda}^{*}$ which assumes values in the interval $(0,1)$.

Summarizing the numerical results one can say that for any value of $\Omega_{\Lambda}^{*}$ the UV cosmologies consist of two scaling regimes and a relatively sharp crossover region near $k, H \approx m_{\mathrm{Pl}}$ corresponding to $x \approx-34.5$ which connects them. At higher $k$-scales the fixed point approximation is valid, at lower scales one has a classical FRW cosmology in which $\Lambda$ can be neglected.

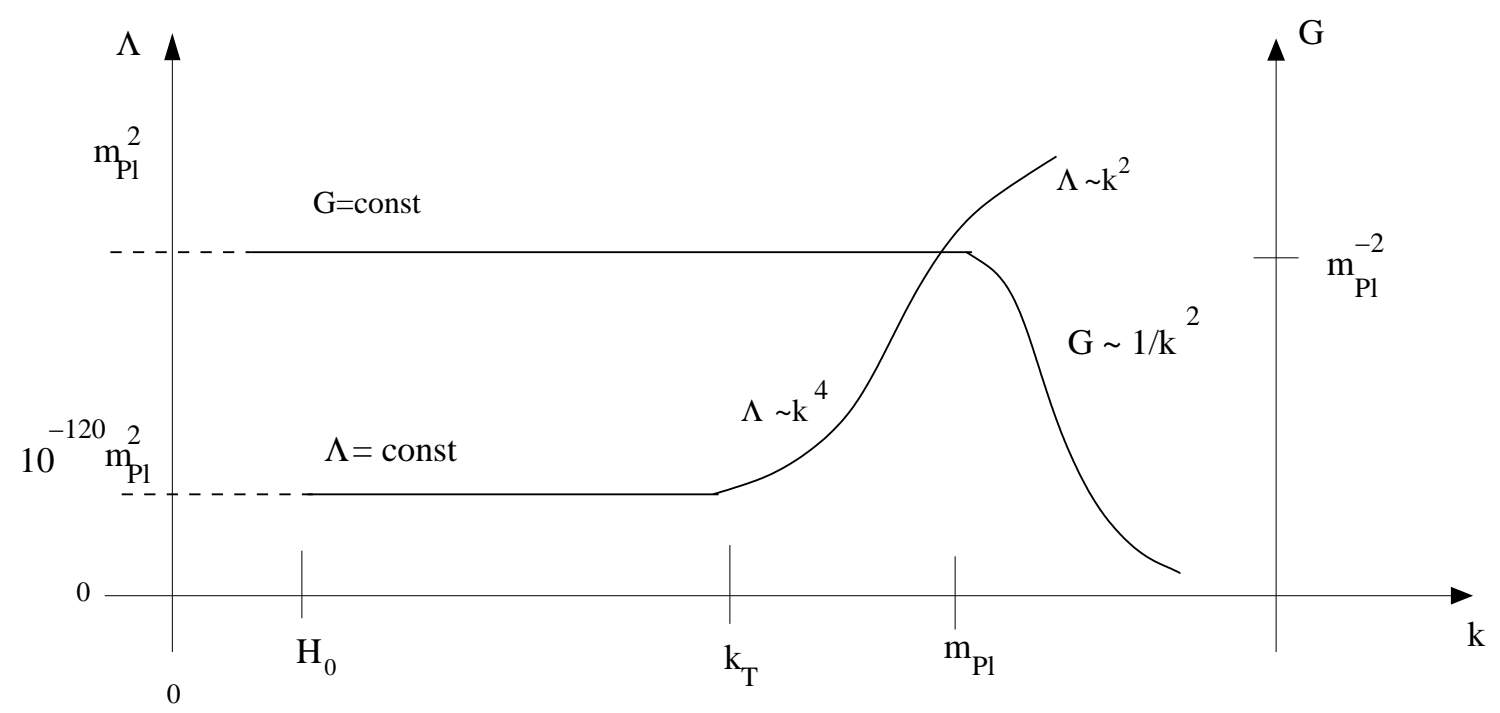

Figure 2: The dimensionful quantities $\Lambda(k)$ and $G(k)$ for the RG trajectory with realistic parameter values.

As an example, Fig.(3) shows the crossover cosmology with $\Omega_{\Lambda}^{*}=0.98$ and $w=1 / 3$. The entropy production rate $\mathscr{P}$ is maximum at $t_{\mathrm{tr}}$ and quickly goes to zero for $t>t_{\mathrm{tr}}$; it is non-zero for all $t<t_{\text {tr }}$. By varying the $\Omega_{\Lambda}^{*}$-value one can check that the early cosmology is indeed described by the NGFP solution (5.1). For the logarithmic $H$ vs. $a$ - plot, for instance, it predicts $\mathscr{U}=-2\left(1-\Omega_{\Lambda}^{*}\right) x$ for $x<-34.4$. The left part of the plot in Fig.3a and its counterparts with different values of $\Omega_{\Lambda}^{*}$ indeed comply with this relation. If $\Omega_{\Lambda}^{*} \in(1 / 2,1)$ we have $\alpha=\left(2-2 \Omega_{\Lambda}^{*}\right)^{-1}>1$ and $a(t) \propto t^{\alpha}$ describes a phase of accelerated power law inflation. 
a)

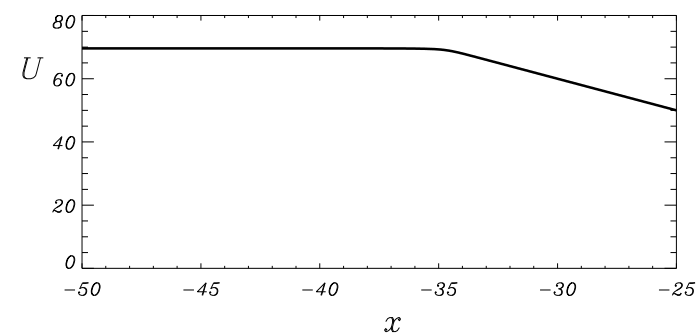

c)

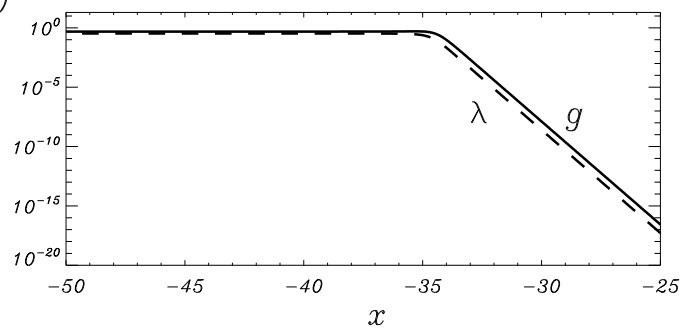

e)

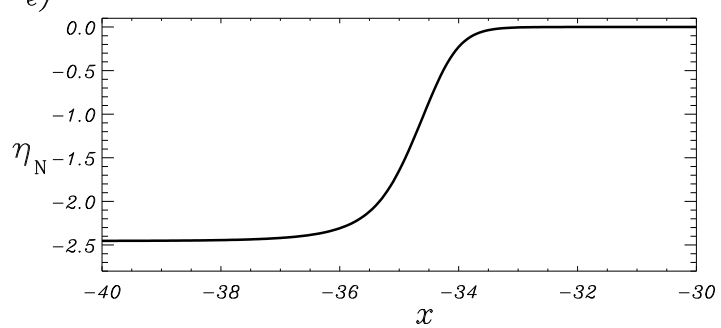

b)

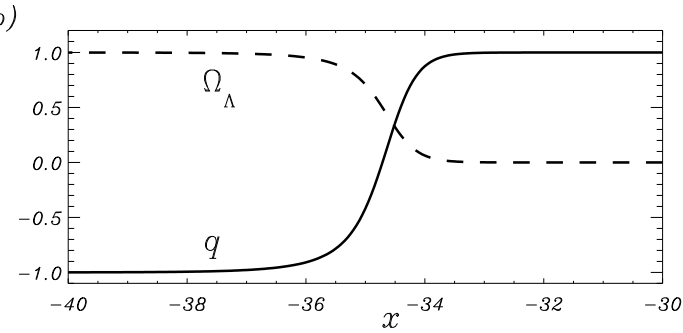

d)

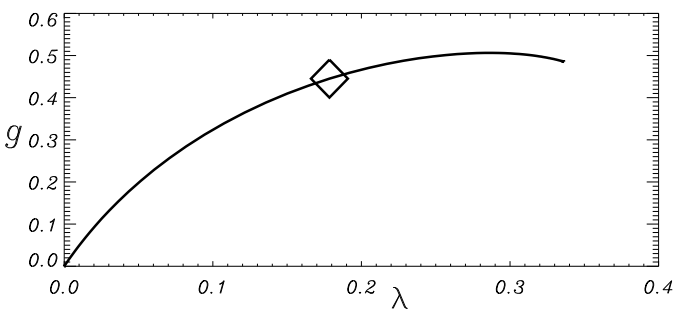

f)

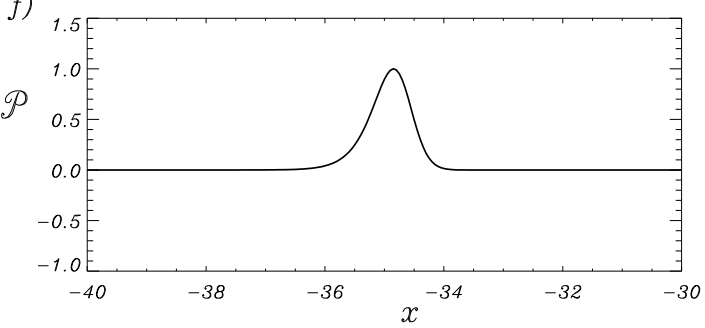

Figure 3: The crossover epoch of the cosmology for $\Omega_{\Lambda}^{*}=0.98$. The plots a), b), c) display the logarithmic Hubble parameter $\mathscr{U}$, as well as $q, \Omega_{\Lambda}, g$ and $\lambda$ as a function of the logarithmic scale factor $x$. A crossover is observed near $x \approx-34.5$. The diamond in plot $\mathrm{d}$ ) indicates the point on the RG trajectory corresponding to this $x$-value. (The lower horizontal part of the trajectory is not visible on this scale.) The plots e) and $\mathrm{f}$ ) show the $x$-dependence of the anomalous dimension and entropy production rate, respectively.

The phase of power law inflation automatically comes to a halt once the RG running has reduced $\Lambda$ to a value where the resulting vacuum energy density no longer can overwhelm the matter energy density.

\section{Inflation in the fixed point regime}

Next we discuss in more detail the epoch of power law inflation which is realized in the NGFP regime if $\Omega_{\Lambda}^{*}>1 / 2$. Since the transition from the fixed point to the classical FRW regime is rather sharp it will be sufficient to approximate the RG improved UV cosmologies by the following caricature : For $0<t<t_{\text {tr }}$, the scale factor behaves as $a(t) \propto t^{\alpha}, \alpha>1$. Here $\alpha=\left(2-2 \Omega_{\Lambda}^{*}\right)^{-1}$ since $w=1 / 3$ will be assumed. Thereafter, for $t>t_{\text {tr }}$, we have a classical, entirely matter-driven expansion $a(t) \propto t^{1 / 2}$.

The transition time $t_{\mathrm{tr}}$ is dictated by the RG trajectory. It leaves the asymptotic scaling regime 
near $k \approx m_{\mathrm{Pl}}$. Hence $H\left(t_{\mathrm{tr}}\right) \approx m_{\mathrm{Pl}}$ and since $\xi=O(1)$ and $H(t)=\alpha / t$ we find the estimate

$$
t_{\mathrm{tr}}=\alpha t_{\mathrm{Pl}}
$$

Here, as always, the Planck mass, time, and length are defined in terms of the value of Newton's constant in the classical regime : $t_{\mathrm{Pl}}=\ell_{\mathrm{Pl}}=m_{\mathrm{Pl}}^{-1}=\bar{G}^{1 / 2}=G_{\text {observed }}^{1 / 2}$. Let us now assume that $\Omega_{\Lambda}^{*}$ is very close to 1 so that $\alpha$ is large: $\alpha \gg 1$. Then (7.1) implies that the transition takes place at a cosmological time which is much later than the Planck time. At the transition the Hubble parameter is of order $m_{\mathrm{Pl}}$, but the cosmological time is in general not of the order of $t_{\mathrm{Pl}}$. Stated differently, the "Planck time" is not the time at which $H$ and the related physical quantities assume Planckian values. The Planck time as defined above is well within the NGFP regime: $t_{\mathrm{Pl}}=t_{\mathrm{tr}} / \alpha \ll t_{\mathrm{tr}}$.

At $t=t_{\text {tr }}$ the NGFP solution is to be matched continuously with a FRW cosmology (with vanishing cosmological constant ). We may use the classical formula $a \propto \sqrt{t}$ for the scale factor, but we must shift the time axis on the classical side such that $a, H$, and then as a result of (4.1) also $\rho$ are continuous at $t_{\mathrm{tr}}$. Therefore $a(t) \propto\left(t-t_{\mathrm{as}}\right)^{1 / 2}$ and $H(t)=\frac{1}{2}\left(t-t_{\mathrm{as}}\right)^{-1}$ for $t>t_{\mathrm{tr}}$. Equating this Hubble parameter at $t=t_{\text {tr }}$ to $H(t)=\alpha / t$, valid in the NGFP regime, we find that the shift $t_{\text {as }}$ must be chosen as $t_{\mathrm{as}}=\left(\alpha-\frac{1}{2}\right) t_{\mathrm{Pl}}=\left(1-\frac{1}{2 \alpha}\right) t_{\mathrm{tr}}<t_{\mathrm{tr}}$. Here the subscript 'as' stands for "apparent singularity". This is to indicate that if one continues the classical cosmology to times $t<t_{\text {tr }}$, it has an initial singularity ("big bang") at $t=t_{\mathrm{as}}$. Since, however, the FRW solution is not valid there nothing special happens at $t_{\mathrm{as}}$; the true initial singularity is located at $t=0$ in the NGFP regime. (See Fig. 4.)

\subsection{Crossing the Hubble radius}

In the NGFP regime $0<t<t_{\text {tr }}$ the Hubble radius $\ell_{H}(t) \equiv 1 / H(t)$, i.e. $\ell_{H}(t)=t / \alpha$, increases linearly with time but, for $\alpha \gg 1$, with a very small slope. At the transition, the slope jumps from $1 / \alpha$ to the value 2 since $H=1 /(2 t)$ and $\ell_{H}=2 t$ in the FRW regime. This behavior is sketched in Fig. 4.

Let us consider some structure of comoving length $\Delta x$, a single wavelength of a density perturbation, for instance. The corresponding physical, i.e. proper length is $L(t)=a(t) \Delta x$ then. In the NGFP regime it has the time dependence $L(t)=\left(t / t_{\mathrm{tr}}\right)^{\alpha} L\left(t_{\mathrm{tr}}\right)$. The ratio of $L(t)$ and the Hubble radius evolves according to $\frac{L(t)}{\ell_{\mathrm{H}}(t)}=\left(\frac{t}{t_{\mathrm{tr}}}\right)^{\alpha-1} \frac{L\left(t_{\mathrm{tr}}\right)}{\ell_{\mathrm{H}}\left(t_{\mathrm{tr}}\right)}$. For $\alpha>1$, i.e. $\Omega_{\Lambda}^{*}>1 / 2$, the proper length of any object grows faster than the Hubble radius. So objects which are of "sub-Hubble" size at early times can cross the Hubble radius and become "super-Hubble" at later times, see Fig. 4.

Let us focus on a structure which, at $t=t_{\mathrm{tr}}$, is $e^{N}$ times larger than the Hubble radius. Before the transition we have $L(t) / \ell_{\mathrm{H}}(t)=e^{N}\left(t / t_{\mathrm{tr}}\right)^{\alpha-1}$. Assuming $e^{N}>1$, there exists a time $t_{N}<t_{\mathrm{tr}}$ at which $L\left(t_{N}\right)=\ell_{\mathrm{H}}\left(t_{N}\right)$ so that the structure considered "crosses" the Hubble radius at the time $t_{N}$. It is given by

$$
t_{N}=t_{\text {tr }} \exp \left(-\frac{N}{\alpha-1}\right)
$$

What is remarkable about this result is that, even with rather moderate values of $\alpha$, one can easily "inflate" structures to a size which is by many $e$-folds larger than the Hubble radius during a very short time interval at the end of the NGFP epoch.

Let us illustrate this phenomenon by means of an example, namely the choice $\Omega_{\Lambda}^{*}=0.98$ used in Fig. 3. Corresponding to $98 \%$ vacuum and $2 \%$ matter energy density in the NGFP regime, this 


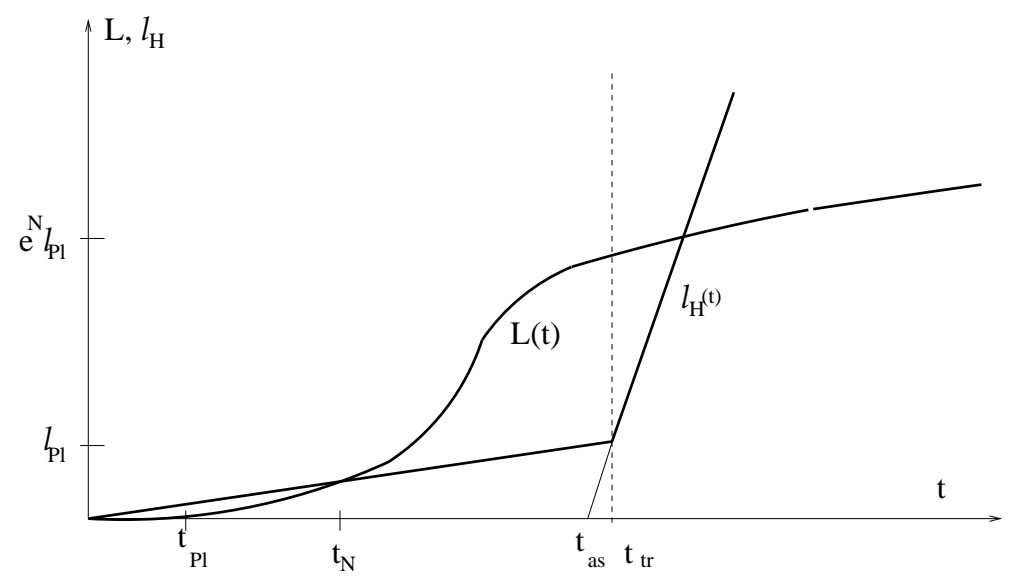

Figure 4: Shown is the proper length $L$ and the Hubble radius as a function of time. The NGFP and FRW cosmologies are valid for $t<t_{\text {tr }}$ and $t>t_{\text {tr }}$, respectively. The classical cosmology has an apparent initial singularity at $t_{a s}$ outside its domain of validity. Structures of size $e^{N} \ell_{\mathrm{Pl}}$ at $t_{\mathrm{tr}}$ cross the Hubble radius at $t_{N}$, a time which can be larger than the Planck time.

value is still "generic" in the sense that $\Omega_{\Lambda}^{*}$ is not fine tuned to equal unity with a precision of many decimal places. It leads to the exponent $\alpha=25$, the transition time $t_{\mathrm{tr}}=25 t_{\mathrm{Pl}}$, and $t_{\mathrm{as}}=24.5 t_{\mathrm{Pl}}$.

The largest structures in the present Universe, evolved backward in time by the classical equations to the point where $H=m_{\mathrm{Pl}}$, have a size of about $e^{60} \ell_{\mathrm{Pl}}$ there. We can use (7.2) with $N=60$ to find the time $t_{60}$ at which those structures crossed the Hubble radius. With $\alpha=25$ the result is $t_{60}=2.05 t_{\mathrm{Pl}}=t_{\mathrm{tr}} / 12.2$. Remarkably, $t_{60}$ is smaller than $t_{\mathrm{tr}}$ by one order of magnitude only. As a consequence, the physical conditions prevailing at the time of the crossing are not overly "exotic" yet. The Hubble parameter, for instance, is only one order of magnitude larger than at the transition: $H\left(t_{60}\right) \approx 12 m_{\mathrm{Pl}}$. The same is true for the temperature; one can show that $T\left(t_{60}\right) \approx 12 T\left(t_{\mathrm{tr}}\right)$ where $T\left(t_{\mathrm{tr}}\right)$ is of the order of $m_{\mathrm{Pl}}$. Note that $t_{60}$ is larger than $t_{\mathrm{Pl}}$.

\subsection{Primordial density fluctuations}

QEG offers a natural mechanism for generating primordial fluctuations during the NGFP epoch. They have a scale free spectrum with a spectral index close to $n=1$. This mechanism is at the very heart of the "asymptotic safety" underlying the non-perturbative renormalizability of QEG. A detailed discussion of this mechanism is beyond the scope of the present review; the reader it referred to $[6,7,40,48]$. Suffice it to say that the quantum mechanical generation of the primordial fluctuations happens on sub-Hubble distance scales. However, thanks to the inflationary NGFP era the modes relevant to cosmological structure formation were indeed smaller than the Hubble radius at a sufficiently early time, for $t<t_{60}$.

\section{RG improved Black Hole spacetimes}

In [30], a "RG-improvement" of the Schwarzschild metric has been performed and the properties of the corresponding "quantum black hole" have been explored. The improvement was based upon the scale dependent ("running") Newton constant $G(k)$ obtained from the exact RG equation 
for gravity describing the scale dependence of the effective average action. In this case the effect of the cosmological constant has been neglected, and the running of $G$ is approximately given by

$$
G(k)=\frac{G_{0}}{1+\omega G_{0} k^{2}}
$$

where $G_{0}$ denotes the laboratory value of Newton's constant, and $\omega$ is a constant. At large distances $(k \rightarrow 0), G(k)$ approaches $G_{0}$, and in the ultraviolet limit $(k \rightarrow \infty)$, it decreases as $G(k) \propto 1 / k^{2}$. This is the fixed point behavior responsible for the conjectured non-perturbative renormalizability of Quantum Einstein Gravity, in the approximation of neglecting the running of the Cosmological Constant.

In the RG improvement scheme of [30] the information about the $k$-dependence of $G$ is exploited in the following way. The starting point is the classical Schwarzschild metric

$$
d s^{2}=-f(r) d t^{2}+f(r)^{-1} d r^{2}+r^{2} d \Omega^{2}
$$

with $d \Omega^{2} \equiv d \theta^{2}+\sin ^{2} \theta d \phi^{2}$ and the classical lapse function $f(r)=1-2 G_{0} M / r \equiv f_{\text {class }}(r)$. The $\mathrm{RG}$ improvement is effected by substituting, in $f_{\text {class }}(r), G_{0}$ by the $r$-dependent Newton constant $G(r) \equiv G(k=k(r))$ which obtains from $G(k)$ via an appropriate "cutoff identification" $k=k(r)$. In flat space the natural choice would be $k \propto 1 / r$. In [30] it was argued that in the Schwarzschild background the correct choice, in leading order at least, is $k(r)=\xi / d(r)$ where $\xi$ is a constant of the order of unity, and $d(r) \equiv \int_{0}^{r} d r^{\prime}\left|f_{\text {class }}\left(r^{\prime}\right)\right|^{-1 / 2}$ is the proper distance from a point with coordinate $r$ to the center of the black hole. While the integral defining $d(r)$ can be evaluated exactly, it is sufficient to use the following approximation which becomes exact for both $r \rightarrow \infty$ and $r \rightarrow 0$ :

$$
d(r)=\left(\frac{r^{3}}{r+\gamma G_{0} M}\right)^{\frac{1}{2}}
$$

The resulting $G(r) \equiv G(k=\xi / d(r))$ reads

$$
G(r)=\frac{G_{0} r^{3}}{r^{3}+\tilde{\omega} G_{0}\left[r+\gamma G_{0} M\right]}
$$

where $\widetilde{\omega} \equiv \omega \xi^{2}$. In these equations the parameter $\gamma$ has the value $\gamma=9 / 2$ if one sets $k=\xi / d(r)$ as above. It turns out, however, that most of the qualitative properties of the improved metric, in particular all those related to the structure of its horizons, are fairly insensitive to the precise value of $\gamma$. In particular, $\gamma=0$ (corresponding to $k=\xi / r$ ) and $\gamma=9 / 2$ where found [30] to lead to rather similar results throughout. For this reason one can adopt the choice $\gamma=0$ in the present paper. It has the advantage that with this choice many calculations can be performed analytically which require a numerical treatment otherwise.

The metric of the RG improved Schwarzschild black hole is given by the line element (8.2) with

$$
f(r)=1-\frac{2 G(r) M}{r}
$$

Let us briefly list its essential features

a) There exists a critical mass value

$$
M_{\mathrm{cr}}=\sqrt{\widetilde{\omega} / G_{0}}=\sqrt{\widetilde{\omega}} m_{\mathrm{Pl}}
$$


such that $f(r)$ has two simple zeros at $r_{-}$and $r_{+}>r_{-}$if $M>M_{\mathrm{cr}}$, one double zero at $r_{+}=r_{-}=$ $\sqrt{\widetilde{\omega} G_{0}}$ if $M=M_{\mathrm{cr}}$, and no zero at all if $M<M_{\mathrm{cr}}$. For $M>M_{\mathrm{cr}}$ the zeros are at

$$
r_{ \pm}=G_{0} M[1 \pm \sqrt{1-\Omega}]
$$

with the convenient abbreviation

$$
\Omega \equiv \frac{M_{\mathrm{cr}}^{2}}{M^{2}}=\widetilde{\omega}\left(\frac{m_{\mathrm{Pl}}}{M}\right)^{2}
$$

The spacetime has an outer horizon at $r_{+}$and in inner (Cauchy) horizon at $r_{-}$. At $M_{\mathrm{cr}}$, the black hole is extremal, the two horizons coincide, and the spacetime is free from any horizon if the mass is sufficiently small, $M<M_{\mathrm{cr}}$.

b) The Bekenstein-Hawking temperature $T_{\mathrm{BH}}=\kappa / 2 \pi$ is given by the surface gravity at the outer horizon, $\kappa=\frac{1}{2} f^{\prime}\left(r_{+}\right)$. Explicitly,

$$
T_{\mathrm{BH}}(M)=\frac{1}{4 \pi G_{0} M} \frac{\sqrt{1-\Omega}}{1+\sqrt{1-\Omega}}=\frac{1}{4 \pi G_{0} M_{\mathrm{cr}}} \frac{\sqrt{\Omega(1-\Omega)}}{1+\sqrt{1-\Omega}}=\frac{M_{\mathrm{cr}}}{4 \pi \widetilde{\omega}} \frac{\sqrt{\Omega(1-\Omega)}}{1+\sqrt{1-\Omega}}
$$

This temperature vanishes for $M \searrow M_{\mathrm{cr}}$, i.e. $\Omega \nearrow 1$, thus motivating the interpretation of the improved Schwarzschild metric with $M=M_{\text {cr }}$ as describing a "cold" remnant of the evaporation process.

c) The energy flux from the black hole, its luminosity $L$, can be estimated using Stefan's law. It is given by $L=\sigma \mathscr{A}(M) T_{\mathrm{BH}}(M)^{4}$ where $\sigma$ is a constant and $\mathscr{A} \equiv 4 \pi r_{+}^{2}$ denotes the area of the outer horizon. With (8.7) and (8.9) we obtain

$$
L(M)=\frac{\sigma M_{\mathrm{cr}}^{2}}{(4 \pi)^{3} \widetilde{\omega}^{2}} \frac{\Omega(1-\Omega)^{2}}{[1+\sqrt{1-\Omega}]^{2}}
$$

For a single massless field with two degrees of freedom one has $\sigma=\pi^{2} / 60$.

\section{The quantum-corrected Vaidya metric}

An important issue is to find a metric which describes the history of an evaporating Schwarzschild black hole and its gravitational field [31]. In the small luminosity limit $(L \rightarrow 0)$ this metric is supposed to reduce to the static metric of the RG improved Schwarzschild spacetime.

By reexpressing the metric (8.2) with the improved lapse function (8.5) in terms of ingoing Eddington-Finkelstein coordinates $(v, r, \theta, \phi)$ it is convenient to trade the Schwarzschild time $t$ for the advanced time coordinate

$$
v=t+r^{\star}, \quad r^{\star} \equiv \int^{r} d r^{\prime} / f\left(r^{\prime}\right)
$$

Here $r^{\star}$ is a generalization of the familiar "tortoise" radial coordinate to which it reduces if $G(r)=$ const. For $G(r) \neq$ const the function $r^{\star}=r^{\star}(r)$ is more complicated, but its explicit form will not be needed here. Eq.(9.1) implies $d v=d t+d r / f(r)$, turning (8.2) with (8.5) into

$$
d s^{2}=-[1-2 G(r) M / r] d v^{2}+2 d v d r+r^{2} d \Omega^{2}
$$



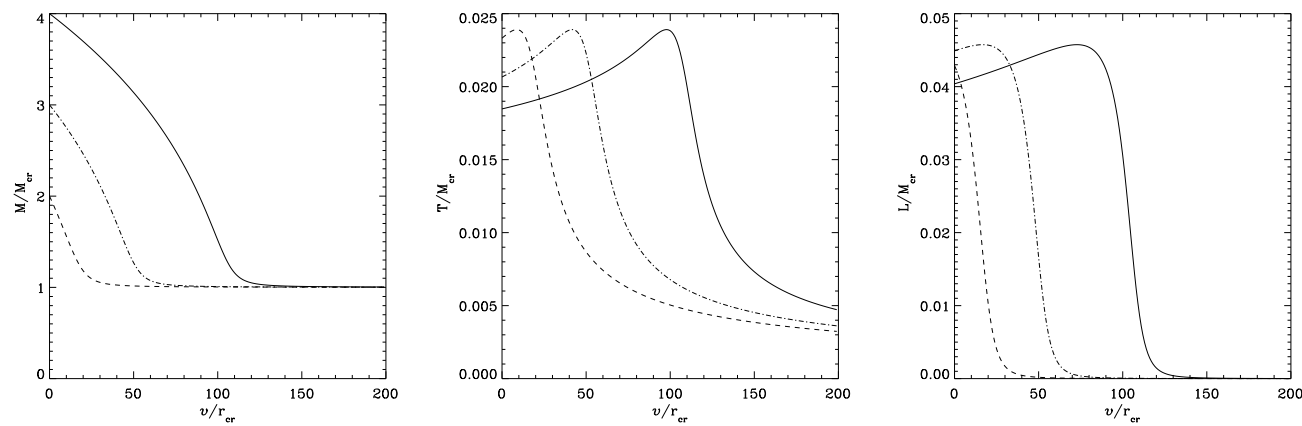

Figure 5: The ratio $M / M_{\mathrm{cr}}$, the Bekenstein-Hawking temperature and the $\mathrm{BH}$ luminosity as a function of $v / r_{\mathrm{cr}}$ for various initial masses, $M / M_{\mathrm{cr}}=1,2,3$, respectively.

Eq.(9.2) is exactly the Schwarzschild metric in Eddington-Finkelstein coordinates, with $G_{0}$ replaced by $G(r)$. It is thus reassuring to see that the two operations, the RG improvement $G_{0} \rightarrow G(r)$ and the change of the coordinate system, can be performed in either order, they "commute".

The thermodynamical properties derived in [30] and summarized in the previous section refer to the metric (9.2). In the exterior of the hole the spacetime is static, and while we can deduce a temperature and a corresponding luminosity from its periodicity in imaginary time (or by computing the surface gravity at $r_{+}$directly) the backreaction of the mass-loss due to the evaporation is not described by (9.2). From the static metric we obtained the mass dependence of the luminosity, $L=L(M)$. Using this information we can compute the mass of the hole as seen by a distant observer at time $v, M(v)$, by solving the differential equation

$$
-\frac{d}{d v} M(v)=L(M(v))
$$

In our case $L(M)$ is given by Eq.(8.10). To first order in the luminosity, the metric which incorporates the effect of the decreasing mass is obtained by replacing the constant $M$ in (9.2) with the $M(v)$ obtained from Eq.(9.3):

$$
d s^{2}=-[1-2 G(r) M(v) / r] d v^{2}+2 d v d r+r^{2} d \Omega^{2}
$$

For $G(r)=$ const, Eq.(9.4) is the Vaidya metric which frequently had been used to explore the influence of the Hawking radiation on the geometry. It is a solution of Einstein's equation $G_{\mu \nu}=$ $8 \pi G_{0} T_{\mu \nu}$ where $T_{\mu \nu}$ describes an inward moving null fluid. In this picture the decrease of $M$ is due to the inflow of negative energy, as it is appropriate if the field whose quanta are radiated off is in the Unruh vacuum.

The metric (9.4) can be regarded as a RG improved Vaidya metric. It encapsulates two different mechanisms whose combined effect can be studied here: the black hole radiance, and the modifications of the spacetime structure due to the quantum gravity effects, the running of $G$ in particular.

It is instructive to ask which energy-momentum tensor $T_{\mu \nu}$ would give rise to the improved Vaidya metric (9.4) according to the classical equation $G_{\mu}{ }^{v}=8 \pi G_{0} T_{\mu}{ }^{\nu}$. Computing the Einstein 
tensor of (9.4) one finds that its only non-zero components are

$$
\begin{aligned}
& T^{v}{ }_{v}=T^{r}{ }_{r}=-\frac{G^{\prime}(r) M(v)}{8 \pi G_{0} r^{2}} \\
& T^{r}{ }_{v}=\frac{G(r) \dot{M}(v)}{8 \pi G_{0} r^{2}} \\
& T^{\theta}{ }_{\theta}=T_{\phi}^{\phi}=-\frac{G^{\prime \prime}(r) M(v)}{16 \pi G_{0} r}
\end{aligned}
$$

Here the prime (dot) denotes a derivative with respect to $r(v)$. The non-zero components (9.5) contain either $r$ - or $v$-derivatives but no mixed terms. The terms with $r$-derivatives of $G$, also present for $M(v)=$ const, describe the vacuum energy density and pressure of the improved Schwarzschild spacetime in absence of radiation effects. Allowing for $M(v) \neq$ const, the new feature is a nonzero component $T^{r}{ }_{v} \neq 0$ which, for $\dot{M}<0$, describes the inflow of negative energy into the black hole.

Taking advantage of the luminosity function $L(M)$, Eq.(8.10), we can solve the differential equation (9.3) numerically and obtain the mass function $M=M(v)$. (We have set $\sigma /(4 \pi)^{3} \widetilde{\omega}=1$ in the numerical calculations in order to reach the almost complete evaporation for $v \approx 200$ in units of $r_{\mathrm{cr}}$.) The result is shown in Fig.(5) for various initial masses, in the domain $v>0$. In fact, for definiteness we assume that the black hole is formed at $v=0$ by the implosion of a spherical null shell. Hence $M(v)$ is given by Fig.(5) together with $M=0$ for $v<0$. We observe that, for any initial mass, $M(v)$ approaches the critical mass $M_{\mathrm{cr}}$ for $v \rightarrow \infty$. This behavior is the most important manifestation of the quantum gravity effects: according to Eq.(8.9), the temperature $T_{\mathrm{BH}}(M)$ goes to zero when $M$ approaches $M_{\mathrm{cr}}$ from above. Hence the luminosity vanishes, too, the evaporation process stops, and $M(v) \approx M_{\mathrm{cr}}$ remains approximately constant at very late times, $v \gg M_{\mathrm{cr}}^{-1}$. In Fig.(5) we also plot the advanced time dependence of the temperature $T_{\mathrm{BH}}(v) \equiv T_{\mathrm{BH}}(M(v))$ and the luminosity $L(v) \equiv L(M(v))$, respectively. They are obtained by inserting the numerical solution of Eq.(9.3) into (8.9)

The global structure of the spacetime is depicted in the conformal diagram in Fig.(6). Region I is a flat spacetime, while at $V=V_{0}(V$ is the Kruskal advanced time coordinate, defined as $V=$ $-\exp (-\kappa v)$ being $\kappa$ the surface gravity of the outer horizon) an imploding null shell is present (strictly speaking it must have a negative tension in order to balance the flux of negative energy on its future side. Region II is the evaporating black hole spacetime. The apparent horizon $\mathrm{AH}$ is a timelike hypersurface which "meets" the event horizon EH at future null infinity in the conformal diagram. The null ray which is tangent to the earliest portion of the apparent horizon $A$ would have been the $\mathrm{EH}$ if the hole were not radiating. The final state of the black hole is an extremal black hole whose inner and outer horizons have the same radius $\left(r=r_{\mathrm{cr}}\right)$ and are located at the event horizon $\mathrm{EH}$ and the inner (Cauchy) horizon $\mathrm{CH}$ in Fig.(6).

It is instructive to compare the areas $\mathscr{A}$ of the various horizons. They are defined by intersecting the EH, AH, and TLS with the incoming null surfaces $v=$ const. Thus $\mathscr{A}_{\mathrm{TLS}}(v)=4 \pi r_{+}(v)^{2}$ and $\mathscr{A}_{\mathrm{EH}}(v)=4 \pi r_{\mathrm{EH}}(v)^{2}$. From Eq.(8.7) we obtain for $\mathscr{A}_{\mathrm{TLS}} \equiv \mathscr{A}_{\mathrm{AH}}$

$$
\mathscr{A}_{\mathrm{TLS}}=4 \pi G_{0}^{2} M^{2}\left[1+\sqrt{1-\left(M_{\mathrm{cr}} / M\right)^{2}}\right]^{2}
$$




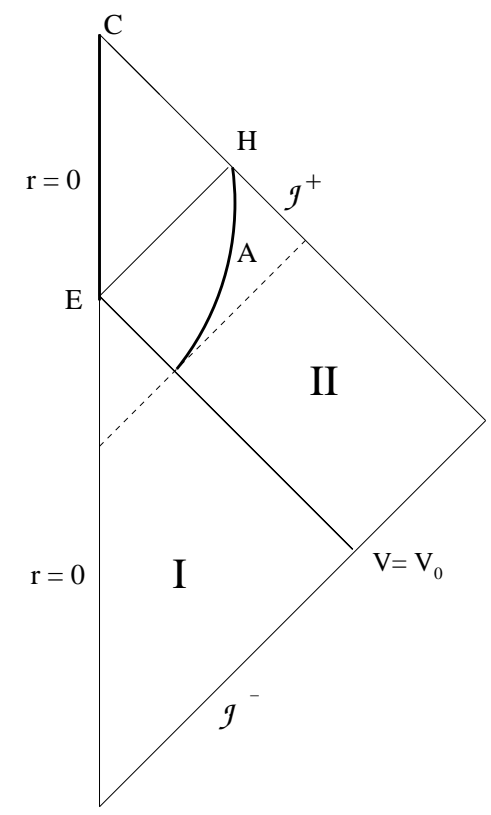

Figure 6: The conformal diagram of the evaporating quantum black hole: region I is a flat spacetime, and region II is the evaporating $\mathrm{BH}$ spacetime, $\mathrm{EH}$ is the event horizon, $\mathrm{CH}$ is the inner (Cauchy) horizon, and $A$ is the apparent horizon.

and for the event horizon implies

$$
\mathscr{A}_{\mathrm{EH}}=\mathscr{A}_{\mathrm{TLS}}\left[1-\frac{4 \sigma}{(4 \pi)^{3} \widetilde{\omega}} \frac{\Omega(1-\Omega)}{1+\sqrt{1-\Omega}}\right]
$$

where a term of second order in $\sigma /(4 \pi)^{3} \widetilde{\omega}$ has been neglected. The difference $\delta \mathscr{A} \equiv \mathscr{A}_{\mathrm{TLS}}-\mathscr{A}_{\mathrm{EH}}$ is given by

$$
\delta \mathscr{A}=\frac{\sigma}{4 \pi^{2}} \ell_{\mathrm{Pl}}^{2}(1-\Omega)[1+\sqrt{1-\Omega}]
$$

During the early stages of the evaporation process, $\delta \mathscr{A} \approx \sigma l_{\mathrm{Pl}}^{2} /\left(2 \pi^{2}\right)=128 \pi B \ell_{\mathrm{Pl}}^{2}$ which coincides with the known result for the Hawking regime, while $\delta \mathscr{A}$ vanishes proportional to $\left(M^{2}-M_{\mathrm{cr}}^{2}\right) \rightarrow 0$ for $v \rightarrow \infty$. It had been emphasized by York [49] that in the Hawking regime he considered, $\delta \mathscr{A}$ is a universal (i.e. $M$ independent) quantity which depends only on $\sigma$, thus counting the degrees of freedom of the field quanta which can be evaporated off. Looking at Eq.(9.8) we see that this universality does not persist beyond the semiclassical approximation.

In conclusion the renormalization group improvement of black hole spacetimes according to Quantum Einstein Gravity leads to concrete predictions on the final state of the evaporation process. Unlike previous studies based on ad hoc modifications of the equation of state of matter at very high (Planckian) densities, or models based on loop quantum gravity, the mass of the remnant can be calculated explicitly: $M_{\mathrm{cr}}=\sqrt{\widetilde{\omega}} \ell_{\mathrm{Pl}}$. Its precise value is determined by the value of $\widetilde{\omega}$ which is a measurable quantity in principle. No naked singularity forms, so that the remnant is a mini-black hole of Planckian size (See also [50] for an approach based on special resummations of higher order graviton loops, and [51] for an "emergent" spacetime approach). 
It is intriguing to note that remnants of this kind of $\mathrm{TeV}$ mini-black holes can have observable signatures at LHC [52-54].

\section{Quantum Gravity at astrophysical distances}

The realistic RG trajectory described in Fig. 1 terminates before the line $\lambda=1 / 2$ as at this point the $\beta$-functions become singular. It is interesting to see this phenomenon in detail, using for instance the proper-time formulation of the flow equation [14],

$$
\begin{aligned}
& \partial_{t} g=\beta_{g}(g, \lambda) \equiv\left[d-2+\eta_{N}\right] g \\
& \partial_{t} \lambda=\beta_{\lambda}(g, \lambda)
\end{aligned}
$$

being $g(k) \equiv k^{d-2} G_{k} \equiv k^{d-2} Z_{N k}^{-1} \bar{G}$ and the dimensionless cosmological constant $\lambda(k) \equiv k^{-2} \bar{\lambda}_{k}$. The anomalous dimension $\eta_{N} \equiv-\partial_{t} \ln Z_{N k}$ is given by

$$
\eta_{N}=8(4 \pi)^{1-\frac{d}{2}}\left[\frac{d(7-5 d)}{24}(1-2 \lambda)^{\frac{d}{2}-m-2}-\frac{d+6}{6}\right] g \frac{\Gamma\left(m+2-\frac{d}{2}\right)}{\Gamma(m+1)}
$$

and the beta-function of $\lambda$ reads

$$
\beta_{\lambda}=-\left(2-\eta_{N}\right) \lambda+4(4 \pi)^{1-\frac{d}{2}}\left[\frac{d(d+1)}{4}(1-2 \lambda)^{\frac{d}{2}-m-1}-d\right] g \frac{\Gamma\left(m+1-\frac{d}{2}\right)}{\Gamma(m+1)}
$$

where $m>1$ is an integer linked to proper-time regulator [14].

The presence of an IR pole is signaling that the Einstein-Hilbert truncation is no longer a consistent approximation to the full flow equation, and most probably a new set of IR-relevant operators is emerging ad $k \rightarrow 0$. The pole is in fact present in any type of cutoff in the EinsteinHilbert truncation and it is due to the presence of negative eigevalues in the spectrum of $\Gamma_{k}^{(2)}$. As discussed in [41] the dynamical origin of these strong IR effect is due to an "instability driven renormalization", a phenomenon well known from many other physical systems [55-57].

In order to illustrate this point let us look at a scalar model in a simple truncation:

$$
\Gamma_{k}[\phi]=\int \mathrm{d}^{4} x\left\{\frac{1}{2} \partial_{\mu} \phi \partial^{\mu} \phi+\frac{1}{2} m^{2}(k) \phi^{2}+\frac{1}{12} \lambda(k) \phi^{4}\right\} .
$$

Here $\phi$ denotes a real, $\mathscr{Z}_{2}$-symmetric scalar field, and the truncation ansatz (10.4) retains only a running mass and $\phi^{4}$-coupling. In a momentum representation we have

$$
\Gamma_{k}^{(2)}=p^{2}+m^{2}(k)+\lambda(k) \phi^{2}
$$

Always assuming that $\lambda>0$, we see that $\Gamma_{k}^{(2)}$ is positive if $m^{2}>0$; but when $m^{2}<0$ it can become negative for $\phi^{2}$ small enough. Of course, the negative eigenvalue for $\phi=0$, for example, indicates that the fluctuations want to grow, to "condense", and thus to shift the field from the "false vacuum" to the true one. This gives rise to the instability induced renormalizations. In fact, the standard $\beta$ functions for $\mathrm{m}^{2}$ and $\lambda$ can be found by inserting (10.5) into the flow equation, taking two and four 
derivatives with respect to $\phi$, respectively, and then setting $\phi=0$ in order to project out $\partial_{t} m^{2}$ and $\partial_{t} \lambda$. As a result, the $\beta$-functions are given by $p$-integrals over (powers of) the propagator

$$
\left[p^{2}+m^{2}(k)+k^{2}\right]^{-1}
$$

In the symmetric phase $\left(m^{2}>0\right)$ this (euclidean!) propagator has no pole, and the resulting $\beta$ functions are relatively small. In the broken phase $\left(m^{2}<0\right)$, however, there is a pole at $p^{2}=$ $-m(k)^{2}-k^{2}$ provided $k^{2}$ is small enough: $k^{2}<\left|m(k)^{2}\right|$. For $k^{2} \searrow\left|m(k)^{2}\right|$ the $\beta$-functions become large and there are strong instability induced renormalizations.

In a reliable truncation, a physically realistic RG trajectory in the spontaneously broken regime will not hit the singularity at $k^{2}=\left|m(k)^{2}\right|$, but rather make $m(k)$ run in precisely such a way that $\left|m(k)^{2}\right|$ is always smaller than $k^{2}$. This requires that

$$
-m(k)^{2} \propto k^{2}
$$

In order to "cure" the singularity, a mass renormalization is necessary in order to evolve a doublewell shaped symmetry breaking classical potential into an effective potential which is convex and has a flat bottom.

Unfortunately the two-parameter truncation (10.4) is too rudimentary for a reliable description of the broken phase. Its RG trajectories actually do run into the singularity. They terminate at a finite scale $k_{\text {term }}$ with $k_{\text {term }}^{2}=\left|m\left(k_{\text {term }}\right)^{2}\right|$ at which the $\beta$-functions diverge. Instead, if one allows for an arbitrary running potential $U_{k}(\phi)$, containing infinitely many couplings, all trajectories can be continued to $k=0$, and for $k \searrow 0$ one finds indeed the quadratic mass renormalization (10.7) [57].

Let us return to gravity now where $\phi$ corresponds to the metric. In the Einstein-Hilbert truncation it suffices to insert the metric corresponding to a sphere $\mathrm{S}^{4}(r)$ of arbitrary radius $r$ into the flow equation in order to disentangle the contributions from the two invariants $\int \mathrm{d}^{4} x \sqrt{g} \propto r^{4}$ and $\int \mathrm{d}^{4} x \sqrt{g} R \propto r^{2}$. Thus we may think of the Einstein-Hilbert flow as being a manifestation of the dynamics of graviton fluctuations on $\mathrm{S}^{4}(r)$. This family of backgrounds, labeled by $r$, is "off-shell" in the sense that $r$ is completely arbitrary and not fixed by Einstein's equation in terms of $\Lambda$.

It is convenient to decompose the fluctuation $h_{\mu \nu}$ on the sphere into irreducible (TT, TL, $\cdots$ ) components [6] and to expand the irreducible pieces in terms of the corresponding spherical harmonics. For $h_{\mu v}$ in the transverse-traceless (TT) sector, the operator $\Gamma_{k}^{(2)}+R_{k}$ equals, up to a positive constant,

$$
-D^{2}+8 r^{-2}+k^{2}-2 \Lambda(k)
$$

with $D^{2} \equiv g^{\mu v} D_{\mu} D_{v}$ the covariant Laplacian acting on TT tensors. The spectrum of $-D^{2}$, denoted $\left\{p^{2}\right\}$, is discrete and positive. Obviously (10.8) is a positive operator if the cosmological constant is negative. In this case there are only stable, bounded oscillations, leading to a mild fluctuation induced renormalization. The situation is very different for $\Lambda>0$ where, for $k^{2}$ sufficiently small, (10.8) has negative eigenvalues, i. e. unstable eigenmodes. In fact, expanding the RHS of the flow equation to orders $r^{2}$ and $r^{4}$ the resulting $\beta$-functions are given by traces (spectral sums) containing the propagator

$$
\left[p^{2}+k^{2}-2 \Lambda(k)\right]^{-1}
$$


The crucial point is that the propagator (10.9) can have a pole when $\Lambda(k)$ is too large and positive. It occurs for $\Lambda(k) \geq k^{2} / 2$, or equivalently $\lambda(k) \geq 1 / 2$, at $p^{2}=2 \Lambda(k)-k^{2}$. Upon performing the $p^{2}$-sum this pole is seen to be responsible for the terms $\propto 1 /(1-2 \lambda)$ and $\ln (1-2 \lambda)$ in the $\beta$-functions which become singular at $\lambda=1 / 2$. The allowed part of the $g$ - $\lambda$-plane $(\lambda<$ $1 / 2$ ) corresponds to the situation $k^{2}>2 \Lambda(k)$ where the singularity is avoided thanks to the large regulator mass. When $k^{2}$ approaches $2 \Lambda(k)$ from above the $\beta$-functions become large and strong renormalizations set in, driven by the modes which would go unstable at $k^{2}=2 \Lambda$.

In this respect the situation is completely analogous to the scalar theory discussed above: Its symmetric phase $\left(m^{2}>0\right)$ corresponds to gravity with $\Lambda<0$; in this case all fluctuation modes are stable and only small renormalization effects occur. Conversely, in the broken phase $\left(m^{2}<0\right)$ and in gravity with $\Lambda>0$, there are modes which are unstable in absence of the IR regulator. They lead to strong IR renormalization effects for $k^{2} \searrow\left|m(k)^{2}\right|$ and $k^{2} \searrow 2 \Lambda(k)$, respectively. The gravitational Type Ia (Type IIIa) trajectories are analogous to those of the symmetric (broken) phase of the scalar model.

In view of the scalar analogy it is a plausible and very intriguing speculation that, for $k \rightarrow 0$, an improved gravitational truncation has a similar impact on the RG flow as it has in the scalar case. There the most important renormalization effect is the running of the mass: $-m(k)^{2} \propto k^{2}$. If gravity avoids the singularity in an analogous fashion the cosmological constant would run proportional to $k^{2}$,

$$
\Lambda(k)=\lambda_{*}^{\mathrm{IR}} k^{2}
$$

with a constant $\lambda_{*}^{\mathrm{IR}}<1 / 2$. In dimensionless units (10.10) reads $\lambda(k)=\lambda_{*}^{\mathrm{IR}}$, i.e. $\lambda_{*}^{\mathrm{IR}}$ is $a$ infrared fixed point of the $\lambda$-evolution. If the behavior (10.10) is actually realized, the renormalized cosmological constant observed at very large distances, $\Lambda(k \rightarrow 0)$, vanishes regardless of its bare value.

The above discussion has thus lead to the conjecture that the IR behavior of the Newton constant and the cosmological constant is regulated by an IR attractive fixed point. Several investigations [38, 58-61] have shown that in this framework a solution of the "cosmic coincidence problem" arises naturally without the introduction of a quintessence field. In particular in the fixed point regime the vacuum energy density $\rho_{\Lambda} \equiv \Lambda / 8 \pi G$ is automatically adjusted so as to equal the matter energy density, i.e. $\Omega_{\Lambda}=\Omega_{\mathrm{M}}=1 / 2$, and that the deceleration parameter approaches $q=-1 / 4$. Moreover, an analysis of the high-redshift SNe Ia data leads to the conclusion that this infrared fixed point cosmology is in good agreement with the observations [60].

More recent works have instead considered the possibility that the "basin of attraction" of the IR fixed point can act already at galactic scale, thus providing an explanation for the galaxy rotation curve without dark matter [41-43,62], but a detailed analysis based on available experimental data is still missing.

In conclusion, although the existence of an infrared fixed point can only be conjectured on the basis of the above argument, the RG cosmologies derived from it are promising candidates to explain the Dark Energy and Dark Matter issue. 


\section{Conclusions}

In these notes some important astrophysical consequences of the Asymptotic Safety Scenario have been reviewed.

In particular it was advocated the point of view that the scale dependence of the gravitational parameters has an impact on the physics of the Universe we live in and, in particular, it has been possible to identify known features of the Universe which could possibly be due to this scale dependence. Three possible candidates for such features are proposed: the entropy carried by the radiation which fills the Universe today, a period of automatic, $\Lambda$-driven inflation that requires no ad hoc inflaton, and the primordial density perturbations.

Moreover, the impact of the leading quantum gravity effects on the dynamics of the Hawking evaporation process of a black hole have also been investigated. Its spacetime structure is described by a renormalization group improved Vaidya metric. Its event horizon, apparent horizon, and timelike limit surface have been obtained taking the scale dependence of Newton's constant into account. The emergence of a quantum ergosphere is discussed. The final state of the evaporation process is a cold, Planck size remnant.

It would be interesting to investigate the possible astrophysical implications of a population of stable Planck size mini-black holes produced in the Early Universe or by the interaction of cosmic rays with the interstellar medium. I hope to address this issue in a subsequent publication.

\section{Acknowledgments}

It is a pleasure to thank Daniel Litim and all the organizers of the Brighton Workshop on Continuum and Lattice Approaches to Quantum Gravity for their cordial hospitality and for creating a stimulating scientific atmosphere. I would also like to thank Martin Reuter for useful comments.

\section{References}

[1] S. Weinberg, Ultraviolet divergences in quantum theories of gravitation, in General Relativity, an Einstein Centenary Survey (S. Hawking and W. Israel, eds.), Cambridge University Press, 1979.

[2] A. Codello, R. Percacci, and C. Rahmede, Investigating the ultraviolet properties of gravity with a Wilsonian renormalization group equation, Annals of Physics 324 (Feb., 2009) 414-469, [0805.2909].

[3] M. Niedermaier and M. Reuter, The asymptotic safety scenario in quantum gravity, Living Reviews in Relativity 9 (Dec., 2006) 5.

[4] M. Reuter, Nonperturbative evolution equation for quantum gravity, 57 (1998) 971-985, [arXiv:hep-th/9605030].

[5] D. Dou and R. Percacci, The running gravitational couplings., Classical and Quantum Gravity 15 (Nov., 1998) 3449-3468, [arXiv: hep-th/9707239].

[6] O. Lauscher and M. Reuter, Ultraviolet fixed point and generalized flow equation of quantum gravity, 65 (Jan., 2002) 025013-+, [arXiv: hep-th/ 0108040 ].

[7] O. Lauscher and M. Reuter, Flow equation of quantum Einstein gravity in a higher-derivative truncation, 66 (July, 2002) 025026-+, [arXiv: hep-th/0205062]. 
[8] O. Lauscher and M. Reuter, Is quantum Einstein gravity nonperturbatively renormalizable?, Classical and Quantum Gravity 19 (Feb., 2002) 483-492, [arXiv : hep-th/0110021].

[9] M. Reuter and F. Saueressig, Renormalization group flow of quantum gravity in the Einstein-Hilbert truncation, 65 (Mar., 2002) 065016-+, [arXiv: hep-th/0110054].

[10] M. Reuter and F. Saueressig, A class of nonlocal truncations in quantum Einstein gravity and its renormalization group behavior, 66 (Dec., 2002) 125001-+, [arXiv: hep-th/0206145].

[11] W. Souma, Non-Trivial Ultraviolet Fixed Point in Quantum Gravity, Progress of Theoretical Physics 102 (July, 1999) 181-195, [arXiv: hep-th/9907027].

[12] R. Percacci and D. Perini, Constraints on matter from asymptotic safety, 67 (Apr., 2003) 081503-+, [arXiv:hep-th/0207033].

[13] D. F. Litim, Fixed Points of Quantum Gravity, Physical Review Letters 92 (May, 2004) 201301-+, [arXiv:hep-th/0312114].

[14] A. Bonanno and M. Reuter, Proper time flow equation for gravity, Journal of High Energy Physics 2 (Feb., 2005) 35-+, [arXiv: hep-th/0410191].

[15] A. Codello and R. Percacci, Fixed Points of Higher-Derivative Gravity, Physical Review Letters 97 (Dec., 2006) 221301-+, [arXiv: hep-th/0607128].

[16] M. Reuter and H. Weyer, The role of background independence for asymptotic safety in Quantum Einstein Gravity, General Relativity and Gravitation 41 (Apr., 2009) 983-1011, [0903.2971].

[17] M. Reuter and H. Weyer, Background independence and asymptotic safety in conformally reduced gravity, 79 (May, 2009) 105005-+, [0801.3287].

[18] P. F. Machado and R. Percacci, Conformally reduced quantum gravity revisited, ArXiv e-prints (Apr., 2009) [0904.2510].

[19] E. Manrique and M. Reuter, Bare vs. Effective Fixed Point Action in Asymptotic Safety: The Reconstruction Problem, ArXiv e-prints (May, 2009) [0905 . 4220].

[20] J. Berges, N. Tetradis, and C. Wetterich, Non-perturbative renormalization flow in quantum field theory and statistical physics, 363 (June, 2002) 223-386, [arXiv: hep-ph/0005122].

[21] W. Dittrich and M. Reuter, Effective Lagrangians in Quantum Electrodynamics, vol. 220. Springer, Berlin, 1985.

[22] O. Lauscher and M. Reuter, Fractal spacetime structure in asymptotically safe gravity, Journal of High Energy Physics 10 (Oct., 2005) 50-+, [arXiv : hep-th/0 508202 ].

[23] M. Reuter and J.-M. Schwindt, A minimal length from the cutoff modes in asymptotically safe quantum gravity, Journal of High Energy Physics 1 (Jan., 2006) 70-+, [arXiv:hep-th/0511021].

[24] M. Reuter and J.-M. Schwindt, Scale-dependent metric and causal structures in Quantum Einstein Gravity, Journal of High Energy Physics 1 (Jan., 2007) 49-+, [arXiv : hep-th/ 0611294 ].

[25] J. Ambjørn, J. Jurkiewicz, and R. Loll, Semiclassical universe from first principles, Physics Letters $B$ 607 (Feb., 2005) 205-213, [arXiv: hep-th/0411152].

[26] J. Ambjørn, J. Jurkiewicz, and R. Loll, Emergence of a 4D World from Causal Quantum Gravity, Physical Review Letters 93 (Sept., 2004) 131301-+, [arXiv : hep-th/ 0404156 ]. 
[27] J. Ambjørn, J. Jurkiewicz, and R. Loll, The Spectral Dimension of the Universe is Scale Dependent, Physical Review Letters 95 (Oct., 2005) 171301-+, [arXiv : hep-th / 0505113 ].

[28] A. Connes, Noncommutative geometry and the standard model with neutrino mixing, Journal of High Energy Physics 11 (Nov., 2006) 81-+, [arXiv : hep-th/0608226].

[29] A. Bonanno, Coarse graining and renormalization group in the Einstein universe, 52 (July, 1995) 969-980, [arXiv:gr-qc/9505051].

[30] A. Bonanno and M. Reuter, Renormalization group improved black hole spacetimes, 62 (Aug., 2000) 043008-+, [arXiv: hep-th/0002196].

[31] A. Bonanno and M. Reuter, Spacetime structure of an evaporating black hole in quantum gravity, 73 (Apr., 2006) 083005-+, [arXiv: hep-th/0602159].

[32] C. Barceló, S. Liberati, and M. Visser, Analogue Gravity, Living Reviews in Relativity 8 (Dec., 2005) 12-+, [arXiv:gr-qc/0505065].

[33] J. Magueijo and L. Smolin, Gravity's rainbow, Classical and Quantum Gravity 21 (Apr., 2004) 1725-1736, [arXiv:gr-qc/0305055].

[34] M. Visser, Emergent rainbow spacetimes: Two pedagogical examples, ArXiv e-prints (Dec., 2007) [0712.0810].

[35] A. B. Migdal, Vacuum polarization in strong non-homogeneous fields, Nuclear Physics B 52 (Jan., 1973) 483-505.

[36] S. G. Matinyan and G. K. Savvidy, Vacuum polarization induced by the intense gauge field, Nuclear Physics B 134 (Mar., 1978) 539-545.

[37] H. Pagels and E. Tomboulis, Vacuum of the quantum Yang-Mills theory and magnetostatics, Nuclear Physics B 143 (Oct., 1978) 485-502.

[38] M. Reuter and F. Saueressig, Nonlocal quantum gravity and the size of the universe, Fortschritte der Physik 52 (June, 2004) 650-654, [arXiv: hep-th/0311056].

[39] M. Reuter and F. Saueressig, From big bang to asymptotic de Sitter: complete cosmologies in a quantum gravity framework, Journal of Cosmology and Astro-Particle Physics 9 (Sept., 2005) 12-+, [arXiv:hep-th/0507167].

[40] A. Bonanno and M. Reuter, Entropy signature of the running cosmological constant, Journal of Cosmology and Astro-Particle Physics 8 (Aug., 2007) 24-+, [0 706.0174 ].

[41] M. Reuter and H. Weyer, Quantum gravity at astrophysical distances?, Journal of Cosmology and Astro-Particle Physics 12 (Dec., 2004) 1-+, [arXiv: hep-th/0410119].

[42] M. Reuter and H. Weyer, Running Newton constant, improved gravitational actions, and galaxy rotation curves, 70 (Dec., 2004) 124028-+, [arXiv: hep-th/0410117].

[43] M. Reuter and H. Weyer, Renormalization group improved gravitational actions: A Brans-Dicke approach, 69 (May, 2004) 104022-+, [arXiv: hep-th/0311196].

[44] A. Bonanno, G. Esposito, and C. Rubano, Arnowitt Deser Misner gravity with variable $G$ and $\Lambda$ and fixed-point cosmologies from the renormalization group, Classical and Quantum Gravity 21 (Nov., 2004) 5005-5016, [arXiv: gr-qc/0403115].

[45] A. Bonanno, G. Esposito, and C. Rubano, Improved Action Functionals in Non-Perturbative Quantum Gravity, International Journal of Modern Physics A 20 (2005) 2358-2363, [arXiv: hep-th/0511188]. 
[46] N. Goldenfeld, Lectures on phase transitions and the renormalization group, . Reading, USA: Addison-Wesley (1992) 394 p. (Frontiers in physics, 85).

[47] R. P. Woodard, Cosmology Is Not a Renormalization Group Flow, Physical Review Letters 101 (Aug., 2008) 081301-+, [0805.3089].

[48] A. Bonanno and M. Reuter, Cosmology of the Planck era from a renormalization group for quantum gravity, 65 (Feb., 2002) 043508-+, [arXiv : hep-th/0106133].

[49] J. W. York, Jr., Dynamical origin of black-hole radiance, 28 (Dec., 1983) 2929-2945.

[50] B. F. L. Ward, Resummed Quantum Gravity, International Journal of Modern Physics D 17 (2008) 627-633, [arXiv: hep-ph/0610232].

[51] G. Salesi and E. di Grezia, Black hole evaporation within a momentum-dependent metric, 79 (May, 2009) 104009-+, [0902.3763].

[52] B. Betz, M. Bleicher, U. Harbach, T. Humanic, B. Koch, and H. Stöcker, Mini Black Holes at the LHC: Discovery Through Di-Jet Suppression, Mono-Jet Emission and a Supersonic Boom in the Quark-Gluon Plasma in ALICE, ATLAS and CMS, ArXiv High Energy Physics - Phenomenology e-prints (June, 2006) [arXiv: hep-ph/0606193].

[53] T. G. Rizzo, Collider production of TeV scale black holes and higher-curvature gravity, Journal of High Energy Physics 6 (June, 2005) 79-+, [arXiv: hep-ph/0503163].

[54] D. F. Litim and T. Plehn, Signatures of Gravitational Fixed Points at the Large Hadron Collider, Physical Review Letters 100 (Apr., 2008) 131301-+, [0707 . 3983].

[55] J. Alexandre, V. Branchina, and J. Polonyi, Instability induced renormalization, Physics Letters B 445 (Jan., 1999) 351-356, [arXiv : cond-mat/9803007].

[56] O. Lauscher, M. Reuter, and C. Wetterich, Rotation symmetry breaking condensate in a scalar theory, 62 (Dec., 2000) 125021-+, [arXiv: hep-th/0006099].

[57] A. Bonanno and G. Lacagnina, Spontaneous symmetry breaking and proper-time flow equations, Nuclear Physics B 693 (Aug., 2004) 36-50, [arXiv : hep-th / 0403176 ].

[58] A. Bonanno and M. Reuter, Cosmology with self-adjusting vacuum energy density from a renormalization group fixed point, Physics Letters B 527 (Feb., 2002) 9-17, [arXiv:astro-ph/0106468].

[59] A. Bonanno and M. Reuter, Cosmological Perturbations in Renormalization Group Derived Cosmologies, International Journal of Modern Physics D 13 (2004) 107-121, [arXiv:astro-ph/0210472].

[60] E. Bentivegna, A. Bonanno, and M. Reuter, Confronting the IR fixed point cosmology with high-redshift observations, Journal of Cosmology and Astro-Particle Physics 1 (Jan., 2004) 1-+, [arXiv:astro-ph/0303150].

[61] A. Bonanno, G. Esposito, C. Rubano, and P. Scudellaro, The accelerated expansion of the universe as a crossover phenomenon, Classical and Quantum Gravity 23 (May, 2006) 3103-3110, [arXiv: astro-ph/0507670].

[62] G. Esposito, C. Rubano, and P. Scudellaro, Spherically symmetric ADM gravity with variable G and $\Lambda_{c}$, ClassicalandQuantumGravity24(Dec., 2007)6255 - -6266, [0709.1403]. 